Supplement of Clim. Past Discuss., 11, 5049-5071, 2015

http://www.clim-past-discuss.net/11/5049/2015/

doi:10.5194/cpd-11-5049-2015-supplement

(C) Author(s) 2015. CC Attribution 3.0 License.

(c) (i)

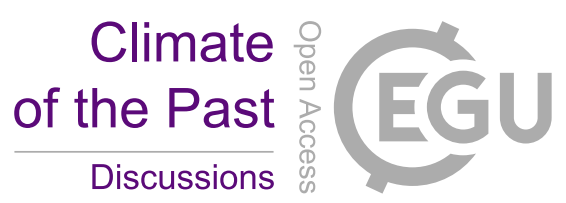

Supplement of

\title{
Late Cretaceous (Late Campanian- \\ Maastrichtian) sea surface temperature record of the Boreal Chalk Sea
}

N. Thibault et al.

Correspondence to: N. Thibault (nt@ign.ku.dk)

The copyright of individual parts of the supplement might differ from the CC-BY 3.0 licence. 


\begin{tabular}{|c|c|c|c|c|c|c|c|c|c|c|c|}
\hline 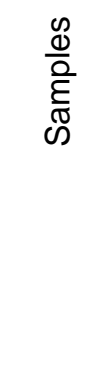 & $\begin{array}{l}\widehat{\underline{\xi}} \\
\text { 劳 } \\
\text { के }\end{array}$ & 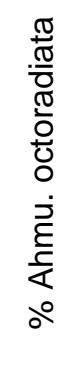 & 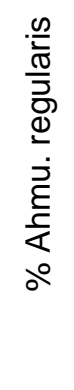 & 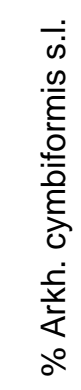 & 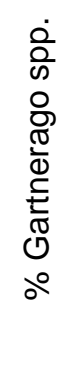 & 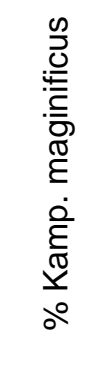 & 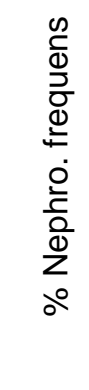 & 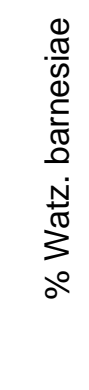 & $E$ & 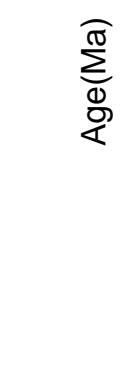 & 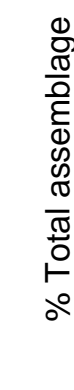 \\
\hline 1475 & -13.28 & 0.5 & 3.0 & 20.8 & 0.2 & 1.7 & 6.8 & 0.6 & 0.108 & 66.155 & 3.5 \\
\hline 8395 & -13.57 & 1.2 & 3.4 & 12.2 & 0.0 & 0.3 & 19.8 & 1.3 & 0.123 & 66.157 & 8.2 \\
\hline 8394 & -13.81 & 0.2 & 1.5 & 10.8 & 0.0 & 0.4 & 18.5 & 0.9 & 0.075 & 66.16 & 2.4 \\
\hline 8393 & -14.07 & 0.9 & 2.5 & 11.9 & 0.0 & 0.9 & 12.6 & 3.0 & 0.173 & 66.162 & 1.7 \\
\hline 1474 & -14.29 & 1.3 & 2.5 & 12.3 & 0.0 & 0.8 & 9.8 & 2.5 & 0.169 & 66.164 & 9.2 \\
\hline 8392 & -14.69 & 0.8 & 3.0 & 9.5 & 0.2 & 1.2 & 6.9 & 6.5 & 0.338 & 66.168 & 8.1 \\
\hline 8391 & -14.94 & 0.9 & 3.1 & 8.6 & 0.0 & 1.4 & 9.7 & 3.8 & 0.248 & 66.171 & 27.6 \\
\hline 1473 & -15.37 & 1.0 & 4.0 & 11.1 & 0.2 & 0.8 & 2.8 & 6.5 & 0.398 & 66.175 & 6.3 \\
\hline 1471 & -17.25 & 1.0 & 2.8 & 7.6 & 0.0 & 1.2 & 7.2 & 5.4 & 0.325 & 66.193 & 5.3 \\
\hline 1469 & -19.00 & 1.2 & 1.4 & 6.9 & 0.3 & 0.7 & 7.5 & .8 & 0.402 & 66.21 & 7.8 \\
\hline 1467 & -21.39 & 1.0 & 1.8 & 12.7 & 0.2 & 1. & 4.7 & 4.3 & 0.238 & 66.232 & 5.8 \\
\hline 1466 & -22.10 & 1.5 & 3.8 & 7.4 & 0.4 & 1.1 & 6.0 & 3.6 & 0.310 & 66.239 & 3.8 \\
\hline 1463 & -24.42 & 1.2 & 3.5 & 5.4 & 0.3 & 0.9 & 10.2 & 5.9 & 0.342 & 66.262 & 27.4 \\
\hline 1462 & -26.62 & 0.7 & 1.8 & 15.4 & 0.9 & 0.4 & 5.0 & 7.7 & 0.299 & 66.283 & 2.0 \\
\hline 1458 & -30.16 & 0.6 & 1.1 & 21.9 & 0.2 & 0.8 & 4.2 & 3.8 & 0.148 & 66.317 & 2.6 \\
\hline 1455 & -32.88 & 0.7 & 1.0 & 6.8 & 0.0 & 2.8 & 3.5 & 2.6 & 0.208 & 66.343 & 7.3 \\
\hline 1452 & -35.42 & 0.7 & 0.2 & 4.7 & 0.0 & 2.8 & 6.3 & 0.9 & 0.075 & 66.367 & 15.6 \\
\hline 1447 & -40.24 & 0.2 & 0.4 & 11.9 & 0.0 & 4.0 & 7.9 & 2.2 & 0.097 & 66.44 & 6.5 \\
\hline 1442 & -45.95 & 0.2 & 0.0 & 11.6 & 0.0 & 5.9 & 4.2 & 2.3 & 0.096 & 66.583 & 24.3 \\
\hline 1432 & -55.90 & 1.0 & 0.2 & 6.4 & 0.0 & 6.2 & 5.7 & 1.0 & 0.060 & 66.831 & 20.5 \\
\hline 1428 & -59.28 & 0.2 & 2.0 & 10.0 & 0.7 & 11.1 & 3.3 & 1.1 & 0.109 & 66.916 & 28.4 \\
\hline 1422 & -64.80 & 0.3 & 1.9 & 6.8 & 0.2 & 3.9 & 12.8 & 0.5 & 0.092 & 67.054 & 26.4 \\
\hline 1417 & -69.22 & 0.6 & 0.6 & 16.3 & 0.2 & 16.1 & 0.0 & 0.4 & 0.027 & 67.164 & 34.0 \\
\hline 1411 & -75.00 & 1.0 & 1.4 & 9.9 & 0.2 & 7.7 & 0.0 & 2.6 & 0.173 & 67.309 & 22.8 \\
\hline 1407 & -79.80 & 1.9 & 0.5 & 9 & 0.2 & 5.7 & 0.9 & 1.2 & 0.081 & 429 & 0.3 \\
\hline 1401 & -85.10 & 1.2 & 0.4 & 4.3 & 1.0 & 2.9 & 20.8 & 2.7 & 0.093 & 67.556 & 3.4 \\
\hline 8180 & -90.34 & 1.2 & 0.6 & 3.0 & 0.9 & 5.3 & 20.5 & 1.4 & 0.060 & 67.655 & 2.9 \\
\hline 1390 & -95.53 & 1.7 & 3.9 & 3.0 & 0.3 & 5.2 & 2.3 & 1.2 & 0.289 & 67.752 & 17.7 \\
\hline 8157 & -97.69 & 1.6 & 0.4 & 4.2 & 0.6 & 2.8 & 8.1 & 0.6 & 0.057 & 67.793 & 18.4 \\
\hline 1386 & -99.43 & 1.1 & 1.6 & 3.8 & 0.3 & 3.6 & 19.4 & 2.5 & 0.128 & 67.826 & 32.4 \\
\hline 5266 & -102.37 & 1.8 & 0.7 & 4.0 & 0.4 & 6.7 & 11.2 & 1.1 & 0.071 & 67.881 & 26.0 \\
\hline 1380 & -104.91 & 0.6 & 0.6 & 7.0 & 0.3 & 3.4 & 24.3 & 0.7 & 0.036 & 67.929 & 36.8 \\
\hline 5620 & -106.37 & 0.2 & 1.3 & 6.3 & 0.7 & 0.9 & 24.7 & 3.4 & 0.125 & 67.957 & 37.5 \\
\hline 1370 & -114.81 & 1.4 & 5.7 & 4.7 & 0.6 & 4.7 & 3.5 & 4.3 & 0.402 & 68.108 & 24.9 \\
\hline 1364 & -120.35 & 1.0 & 2.8 & 18.9 & 0.5 & 9.0 & 0.0 & 6.3 & 0.238 & 68.191 & 38.6 \\
\hline 1358 & -125.85 & 1.9 & 6.7 & 9.3 & 0.6 & 9.1 & 0.0 & 3.7 & 0.331 & 68.274 & 31.3 \\
\hline 1353 & -130.73 & 0.5 & 4.0 & 9.4 & 0.5 & 15.9 & 1.4 & 1.4 & 0.163 & 68.348 & 33.3 \\
\hline 1348 & -135.13 & 2.7 & 6.4 & 6.2 & 0.1 & 4.3 & 0.0 & 10.1 & 0.552 & 68.414 & 9.8 \\
\hline 1337 & -145.25 & 2.7 & 4.2 & 5.3 & 0.5 & 7.7 & 0.0 & 2.1 & 0.279 & 68.566 & $2<$ \\
\hline
\end{tabular}




\begin{tabular}{|c|c|c|c|c|c|c|c|c|c|c|c|}
\hline 1327 & -154.55 & 1.0 & 4.3 & 8.1 & 0.2 & 6.7 & 0.0 & 3.1 & 0.316 & 68.706 & 23.3 \\
\hline 1316 & -164.80 & 1.8 & 2.5 & 5.1 & 0.5 & 6.9 & 0.0 & 3.1 & 0.282 & 68.860 & 20.0 \\
\hline 1310 & -170.50 & 2.1 & 4.3 & 3.0 & 0.4 & 7.6 & 0.0 & 5.9 & 0.435 & 68.945 & 23.3 \\
\hline 1305 & -175.22 & 0.3 & 9.5 & 7.5 & 0.3 & 2.9 & 0.0 & 4.5 & 0.559 & 69.016 & 24.9 \\
\hline 1300 & -179.79 & 0.4 & 0.0 & 10.8 & 0.4 & 5.6 & 0.0 & 2.2 & 0.111 & 69.085 & 19.4 \\
\hline 1294 & -185.10 & 2.6 & 4.9 & 3.1 & 0.5 & 3.7 & 0.0 & 0.5 & 0.354 & 69.165 & 3 \\
\hline 1287 & -191.24 & 0.2 & 0.7 & 0.7 & 0.0 & 8.5 & 0.0 & 2.2 & 0.232 & 69.257 & \\
\hline 1283 & -194.84 & 2.1 & 2.3 & 1.8 & 1.2 & 3.7 & 0.0 & 0.9 & 0.265 & 69.311 & \\
\hline 1272 & -205.38 & 1.0 & 5.6 & 2.0 & 1.3 & 6.2 & 0.0 & 2.0 & 0.420 & 69.488 & \\
\hline 1262 & -214.60 & 3.0 & 2.2 & 6.1 & 0.2 & 6.7 & 0.0 & 1.1 & 0.169 & 69.673 & \\
\hline 1257 & -219.29 & 0.0 & 0.0 & 7.6 & 0.7 & 28.1 & 0.0 & 1.8 & 0.048 & 69.767 & \\
\hline 1250 & -225.58 & 1.6 & 0.0 & 10.0 & 2.0 & 17.9 & 0.0 & 0.7 & 0.021 & 69.893 & \\
\hline 1240 & -234.89 & 0.4 & 0.0 & 16.0 & 0.4 & 15.8 & 0.0 & 0.4 & 0.013 & 70.079 & \\
\hline 1229 & -244.95 & 1.4 & 2.6 & 4.0 & 0.8 & 22.3 & 0.0 & 1.2 & 0.118 & 70.281 & \\
\hline 1218 & -255.20 & 2.7 & 0.2 & 15.4 & 0.5 & 14.0 & 0.0 & 1.0 & 0.036 & 70.486 & \\
\hline 1207 & -265.49 & 2.8 & 0.6 & 4.2 & 0.8 & 4.8 & 0.0 & 0.2 & 0.060 & 70.693 & \\
\hline 1203 & -269.11 & 0.4 & 0.5 & 4.6 & 0.4 & 15.3 & 0.0 & 0.7 & 0.057 & 70.765 & 1.9 \\
\hline 1196 & -275.54 & 3.1 & 0.6 & 11.3 & 1.2 & 9.1 & 0.0 & 0.4 & 0.040 & 70.894 & 0 \\
\hline 1186 & -284.81 & 1.7 & 1.7 & 11.0 & 1.7 & 5.1 & 0.0 & 0.7 & 0.111 & 71.080 & 22.0 \\
\hline 1175 & -295.04 & 1.6 & 1.1 & 5.2 & 2.7 & 3.3 & 0.0 & 1.4 & 0.165 & 71.285 & 15.2 \\
\hline 1170 & -299.63 & 0.7 & 0.2 & 1.7 & 0.4 & 11.7 & 0.0 & 3.3 & 0.196 & 71.377 & 18.0 \\
\hline 1164 & -305.29 & 2.3 & 1.7 & 1.7 & 1.2 & 6.8 & 0.0 & 1.0 & 0.188 & 71.490 & 4.9 \\
\hline 7398 & -310.10 & 2.1 & 7.8 & 2.3 & 0.0 & 2.7 & 0.0 & 0.7 & 0.544 & 669 & 5.6 \\
\hline 1153 & -315.41 & 3.0 & 1.7 & 0.9 & 1.1 & 5.9 & 0.0 & 5.0 & 0.383 & 72.007 & 17.6 \\
\hline 7372 & $-31 c$ & 1.0 & 0.5 & 0.5 & 0.7 & 1.7 & 0.0 & 23.7 & 0.863 & 72 & 8.0 \\
\hline 1141 & -325.77 & 1.3 & 0.7 & 3.4 & 2.9 & 5.4 & 0.0 & 8.3 & 0.408 & 72.294 & 21.9 \\
\hline 1131 & -335.06 & 1.2 & 1.0 & 0.6 & 4.3 & 2.1 & 0.0 & 5.2 & 0.429 & 72.459 & 14.4 \\
\hline 1125 & -340.38 & 0.0 & 0.0 & 0.2 & 0.9 & 3.0 & 0.0 & 2.3 & 0.367 & 72.553 & \\
\hline 1119 & -345.85 & 0.2 & 0.9 & 0.9 & 2.8 & 2.1 & 0.0 & 6.7 & 0.559 & 72.650 & 13.7 \\
\hline 1114 & -350.72 & 0.2 & 0.5 & 4.9 & 4.2 & 3.0 & 0.0 & 3.7 & 0.254 & 72.736 & 16. \\
\hline 1109 & -355.34 & 1.2 & 5.1 & 2.0 & 2.7 & 1.3 & 0.0 & 4.0 & 0.560 & 72.818 & 16.3 \\
\hline 1098 & -365.65 & 0.0 & 2.4 & 0.2 & 0.2 & 0.9 & 0.0 & 5.1 & 0.850 & 72.967 & 8.8 \\
\hline 1087 & -375.86 & 0.0 & 1.5 & 1.1 & 0.4 & $1 . \varepsilon$ & 0.0 & 8.4 & 0.750 & 73.036 & 13.2 \\
\hline 1083 & -379.61 & 1.7 & 1.2 & 0.3 & 0.2 & 1.2 & 0.0 & 10.7 & 0.778 & 73.062 & 5.3 \\
\hline 1077 & -385.18 & 0.5 & 1.4 & 0.2 & 2.3 & 1.6 & 0.0 & 6.3 & 0.623 & 73.100 & 12. \\
\hline 1066 & -395.40 & 1.4 & 1.6 & 0.4 & 2.0 & 2.0 & 0.0 & 10.7 & 0.678 & 73.169 & 18 \\
\hline 1055 & -405.77 & 3.4 & 1.1 & 1.6 & 1.1 & 4.5 & 0.0 & 12.7 & 0.565 & 73.240 & 24.5 \\
\hline 1044 & -415.78 & 0.4 & 1.4 & 0.4 & 1.6 & 4.1 & 0.0 & 16.6 & 0.736 & 73.342 & 24.4 \\
\hline 1034 & -425.24 & 1.0 & 4.0 & 1.4 & 1.4 & 4.3 & 0.0 & 13.6 & 0.685 & 73.475 & 25.7 \\
\hline 1029 & -429.71 & 0.0 & 1.4 & 0.7 & 0.7 & 2.8 & 0.0 & 9.5 & 0.719 & 73.554 & 15.2 \\
\hline 7086 & -430.25 & 0.0 & 0.5 & 1.1 & 0.2 & 2.0 & 0.0 & 15.8 & 0.828 & 73.562 & 19.7 \\
\hline 7076 & -432.88 & 0.4 & 1.7 & 0.9 & 0.4 & 1.5 & 0.0 & 14.0 & 0.830 & 73.637 & 18. \\
\hline 7072 & -433.97 & 0.0 & 2.4 & 0.7 & 1.5 & 2.2 & 0.0 & 8.6 & 0.714 & 73.672 & 15 \\
\hline 7070 & -434.69 & 1.7 & 3.3 & 0.9 & 4.3 & 1.9 & 0.0 & 9.7 & 0.598 & 73.695 & 21 \\
\hline 1023 & -435.42 & 0.0 & 3.4 & 0.2 & 1.6 & 2.3 & 0.0 & 10.7 & 0.775 & 73.718 & 18 \\
\hline 1017 & -441.13 & 0.0 & 0.7 & 2.6 & 0.5 & 4.6 & 0.0 & 7.8 & 0.528 & 73.900 & 16 \\
\hline 011 & -446.67 & 0.7 & 4.2 & 0.9 & 0.0 & 5.1 & 0.0 & 3.0 & 0.517 & 74.076 & 14 \\
\hline 1007 & -450.48 & 0.0 & 0.0 & 2.8 & 1.4 & 7.0 & 0.0 & 2.6 & 0.186 & 74.198 & \\
\hline
\end{tabular}




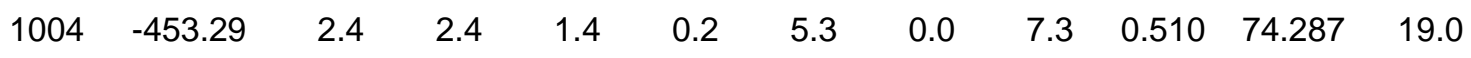

Table S1: Relative abundances of relevant calcareous nannofossil palaeoecological markers in the Stevns-1 core.

\begin{tabular}{|c|c|c|c|c|}
\hline Sample & $\operatorname{depth}(m)$ & $\delta 13 C(\mathrm{PDB})$ & $\delta 180(\mathrm{PDB})$ & Age_(Ma) \\
\hline 8422 & -0.24 & 0.78 & -2.04 & \\
\hline 1485 & -0.92 & 0.74 & -2.02 & \\
\hline 8421 & -1.09 & 0.92 & -2.16 & \\
\hline 8420 & -1.37 & 0.96 & -2.09 & \\
\hline 1484 & -1.63 & 0.86 & -2.09 & \\
\hline 8419 & -2.09 & 0.91 & -1.95 & \\
\hline 8418 & -2.33 & 0.85 & -1.81 & \\
\hline 8417 & -2.58 & 0.78 & -1.82 & \\
\hline 1483 & -2.84 & 0.83 & -2.06 & \\
\hline 8416 & -5.2 & 0.84 & -2.08 & \\
\hline 8415 & -5.44 & 0.85 & -1.79 & \\
\hline 8414 & -5.69 & 0.76 & -1.74 & \\
\hline 1482 & -5.94 & 0.89 & -1.90 & . \\
\hline 8413 & -6.16 & 0.89 & -1.59 & \\
\hline 8412 & -6.45 & 1.09 & -1.74 & \\
\hline 8411 & -6.7 & 1.08 & -1.93 & \\
\hline 1481 & -6.94 & 1.08 & -1.87 & . \\
\hline 8410 & -7.37 & 1.07 & -1.61 & \\
\hline 8409 & -7.62 & 1.17 & -1.47 & \\
\hline 1480 & -7.85 & 1.01 & -1.74 & \\
\hline 8408 & -8 & 1.05 & -1.81 & \\
\hline 8407 & -8.25 & 1.16 & -1.58 & \\
\hline 1479 & -8.5 & 1.06 & -1.50 & \\
\hline 8406 & -9.92 & 1.16 & -2.07 & \\
\hline 8405 & -10.17 & 1.25 & -1.70 & \\
\hline 8404 & -10.42 & 1.24 & -1.58 & \\
\hline 1478 & -10.65 & 1.20 & -1.81 & \\
\hline 8403 & -10.82 & 1.30 & -1.47 & \\
\hline 8402 & -11.07 & 1.31 & -1.61 & \\
\hline 8401 & -11.34 & 1.38 & -2.00 & \\
\hline 1477 & -11.55 & 1.32 & -1.78 & \\
\hline 8400 & -11.68 & 1.25 & -1.81 & \\
\hline 8399 & -11.94 & 1.37 & -1.93 & \\
\hline 8398 & -12.27 & 1.38 & -1.86 & \\
\hline 1476 & -12.47 & 1.40 & -1.61 & \\
\hline 8397 & -12.77 & 1.41 & -1.34 & \\
\hline $\mathrm{K}-\mathrm{Pg}$ & -12.8 & & & 66.150 \\
\hline 8396 & -13.01 & 1.46 & -1.11 & 66.152 \\
\hline 1475 & -13.28 & 1.36 & -1.18 & 66.155 \\
\hline 8395 & -13.57 & 1.41 & -1.47 & 66.157 \\
\hline 8394 & -13.8 & 1.44 & -1.40 & 66.1 \\
\hline
\end{tabular}




$\begin{array}{lrlll}8393 & -14.07 & 1.63 & -1.49 & 66.162 \\ 1474 & -14.29 & 1.53 & -1.37 & 66.164 \\ 8392 & -14.69 & 1.64 & -1.59 & 66.168 \\ 8391 & -14.94 & 1.60 & -1.54 & 66.171 \\ 1473 & -15.37 & 1.34 & -1.59 & 66.175 \\ 8390 & -15.55 & 1.46 & -1.28 & 66.176 \\ 8389 & -15.8 & 1.50 & -1.52 & 66.179 \\ 8388 & -16.05 & 1.59 & -1.59 & 66.181 \\ 1472 & -16.24 & 1.67 & -1.16 & 66.183 \\ 8387 & -16.52 & 1.66 & -1.95 & 66.186 \\ 8386 & -16.76 & 1.60 & -1.39 & 66.188 \\ 8385 & -16.99 & 1.67 & -1.73 & 66.190 \\ 1471 & -17.25 & 1.55 & -1.26 & 66.193 \\ 8384 & -17.7 & 1.56 & -1.40 & 66.197 \\ 8383 & -17.96 & 1.64 & -1.61 & 66.200 \\ 1470 & -18.34 & 1.63 & -1.39 & 66.203 \\ 8382 & -18.53 & 1.60 & -1.44 & 66.205 \\ 8381 & -18.8 & 1.67 & -1.46 & 66.208 \\ 1469 & -19 & 1.61 & -1.45 & 66.210 \\ 8380 & -19.49 & 1.65 & -1.38 & 66.214 \\ 8379 & -19.73 & 1.60 & -1.79 & 66.217 \\ 1468 & -20.04 & 1.54 & -1.19 & 66.220 \\ 8378 & -20.12 & 1.54 & -1.10 & 66.220 \\ 8377 & -20.37 & 1.65 & -1.34 & 66.223 \\ 8376 & -20.73 & 1.55 & -1.20 & 66.226 \\ 8375 & -20.97 & 1.71 & -1.32 & 66.228 \\ 8374 & -21.21 & 1.65 & -1.23 & 66.231 \\ 8373 & -21.34 & 1.72 & -1.33 & 66.232 \\ 1467 & -21.39 & 1.61 & -1.38 & 66.232 \\ 8372 & -21.59 & 1.72 & -0.85 & 66.234 \\ 8371 & -21.86 & 1.76 & -1.41 & 66.237 \\ 1466 & -22.1 & 1.63 & -1.47 & 66.239 \\ 8370 & -22.3 & 1.65 & -0.92 & 66.241 \\ 8369 & -22.57 & 1.66 & -1.44 & 66.244 \\ 1465 & -22.84 & 1.64 & -1.34 & 66.246 \\ 8368 & -23.11 & 1.75 & -1.07 & 66.249 \\ 8367 & -23.36 & 1.63 & -0.71 & 66.251 \\ 1464 & -23.59 & 1.73 & -1.21 & 66.254 \\ 8366 & -23.87 & 1.71 & -1.34 & 66.256 \\ 8365 & -24.16 & 1.64 & -1.11 & 66.259 \\ 1463 & -24.42 & 1.63 & -1.21 & 66.262 \\ 4029 & -24.45 & 1.52 & -1.38 & 66.262 \\ 8364 & -24.66 & 1.68 & -1.01 & 66.264 \\ 8363 & -26.1 & 1.66 & -1.36 & 66.278 \\ 8362 & -26.35 & 1.69 & -0.91 & 66.280 \\ 1462 & -26.62 & 1.67 & -1.64 & 66.283 \\ 8361 & -26.9 & 1.68 & -1.69 & 66.285 \\ 1461 & -27.22 & 1.67 & -1.15 & 66.288\end{array}$




$\begin{array}{lrlll}8360 & -27.4 & 1.66 & -1.64 & 66.290 \\ 8359 & -27.86 & 1.72 & -1.09 & 66.295 \\ 8358 & -28.12 & 1.71 & -1.25 & 66.297 \\ 8357 & -28.37 & 1.80 & -1.09 & 66.300 \\ 1460 & -28.59 & 1.68 & -1.25 & 66.302 \\ 8356 & -28.87 & 1.77 & -1.18 & 66.304 \\ 8355 & -28.98 & 1.75 & -1.12 & 66.305 \\ 8354 & -29.2 & 1.80 & -1.56 & 66.307 \\ 1459 & -29.45 & 1.66 & -1.26 & 66.310 \\ 8353 & -29.49 & 1.70 & -1.33 & 66.310 \\ 8352 & -29.74 & 1.70 & -1.25 & 66.313 \\ 8351 & -29.98 & 1.84 & -0.64 & 66.315 \\ 1458 & -30.16 & 1.62 & -1.13 & 66.317 \\ 8350 & -30.47 & 1.61 & -1.31 & 66.320 \\ 8349 & -30.72 & 1.59 & -1.72 & 66.322 \\ 1457 & -30.98 & 1.58 & -1.22 & 66.325 \\ 8348 & -31.35 & 1.72 & -1.34 & 66.328 \\ 8347 & -31.57 & 1.73 & -1.25 & 66.330 \\ 1456 & -31.91 & 1.64 & -1.21 & 66.334 \\ 8346 & -32.05 & 1.77 & -1.40 & 66.335 \\ 8345 & -32.37 & 1.78 & -1.44 & 66.338 \\ 8344 & -32.69 & 1.81 & -1.34 & 66.341 \\ 1455 & -32.88 & 1.59 & -1.07 & 66.343 \\ 8343 & -32.99 & 1.72 & -1.17 & 66.344 \\ 1454 & -33.24 & 1.63 & -1.22 & 66.346 \\ 8342 & -33.73 & 1.78 & -1.31 & 66.351 \\ 8341 & -33.96 & 1.79 & -0.94 & 66.353 \\ 8340 & -34.28 & 1.84 & -1.13 & 66.356 \\ 1453 & -34.53 & 1.79 & -1.24 & 66.359 \\ 8339 & -34.73 & 1.73 & -0.96 & 66.361 \\ 8338 & -34.96 & 1.81 & -1.12 & 66.363 \\ 8337 & -35.27 & 1.85 & -0.90 & 66.366 \\ 1452 & -35.42 & 1.76 & -0.90 & 66.367 \\ 8336 & -35.67 & 1.79 & -0.85 & 66.370 \\ 8335 & -35.92 & 1.70 & -1.01 & 66.372 \\ 8334 & -36.16 & 1.67 & -1.01 & 66.374 \\ 1451 & -36.42 & 1.67 & -1.01 & 66.377 \\ 1450 & -36.9 & 1.66 & -1.10 & 66.381 \\ 8333 & -37.11 & 1.73 & -1.16 & 66.383 \\ 8332 & -37.63 & 1.80 & -1.12 & 66.388 \\ 8331 & -37.88 & 1.68 & -0.85 & 66.391 \\ 8330 & -38.13 & 1.60 & -1.13 & 66.393 \\ 1449 & -38.37 & 1.57 & -1.29 & 66.396 \\ 8329 & -38.52 & 1.65 & -1.04 & 66.397 \\ 8328 & -38.83 & 1.62 & -1.08 & 66.405 \\ 1448 & -39.07 & 1.56 & -1.04 & 66.411 \\ 8327 & -39.07 & 1.59 & -1.44 & 66.411 \\ 8326 & -39.52 & 1.51 & -0.91 & 66.422\end{array}$




$\begin{array}{lrlll}8325 & -39.77 & 1.55 & -0.97 & 66.428 \\ 8324 & -40.02 & 1.61 & -1.09 & 66.434 \\ 1447 & -40.24 & 1.54 & -1.30 & 66.440 \\ 8323 & -40.48 & 1.59 & -0.98 & 66.446 \\ 8322 & -40.75 & 1.45 & -0.91 & 66.453 \\ 1446 & -41.15 & 1.40 & -1.25 & 66.463 \\ 8321 & -41.2 & 1.59 & -1.37 & 66.464 \\ 8320 & -41.45 & 1.50 & -1.26 & 66.470 \\ 8319 & -41.7 & 1.50 & -0.82 & 66.476 \\ 1445 & -41.93 & 1.57 & -1.08 & 66.482 \\ 8318 & -42.37 & 1.51 & -0.79 & 66.493 \\ 8317 & -42.63 & 1.58 & -1.13 & 66.500 \\ 8316 & -42.89 & 1.56 & -0.83 & 66.506 \\ 1444 & -43.1 & 1.47 & -0.86 & 66.511 \\ 8315 & -43.27 & 1.67 & -1.42 & 66.516 \\ 8314 & -43.49 & 1.49 & -0.76 & 66.521 \\ 8313 & -43.74 & 1.67 & -1.14 & 66.527 \\ 1443 & -43.98 & 1.66 & -1.49 & 66.533 \\ 4027 & -44.15 & 1.70 & -0.90 & 66.538 \\ 8312 & -45.2 & 1.67 & -1.63 & 66.564 \\ 8311 & -45.48 & 1.65 & -0.48 & 66.571 \\ 8310 & -45.73 & 1.69 & -0.97 & 66.577 \\ 1442 & -45.95 & 1.59 & -1.09 & 66.583 \\ 1441 & -46.26 & 1.68 & -1.19 & 66.590 \\ 8309 & -46.64 & 1.88 & -1.15 & 66.600 \\ 8308 & -46.88 & 1.86 & -0.96 & 66.606 \\ 8307 & -47.18 & 1.84 & -1.03 & 66.613 \\ 8306 & -47.41 & 1.82 & -0.95 & 66.619 \\ 8305 & -47.66 & 1.80 & -0.97 & 66.625 \\ 1440 & -47.92 & 1.71 & -1.05 & 66.632 \\ 8304 & -48.07 & 1.81 & -0.77 & 66.636 \\ 8303 & -48.33 & 1.85 & -0.94 & 66.642 \\ 8302 & -48.58 & 1.75 & -0.74 & 66.648 \\ 1439 & -48.74 & 1.89 & -0.85 & 66.652 \\ 8301 & -48.77 & 1.93 & -1.03 & 66.653 \\ 8300 & -49.02 & 1.93 & -0.88 & 66.659 \\ 8299 & -49.27 & 1.86 & -1.00 & 66.666 \\ 1438 & -49.54 & 1.84 & -1.23 & 66.672 \\ 8298 & -49.7 & 1.77 & -0.84 & 66.676 \\ 8297 & -49.96 & 1.88 & -0.94 & 66.683 \\ 1437 & -50.52 & 1.93 & -1.07 & 66.697 \\ 8295 & -50.8 & 1.90 & -0.92 & 66.704 \\ 8294 & -51.15 & 1.84 & -0.94 & 66.713 \\ 1436 & -51.38 & 1.94 & -1.18 & 66.718 \\ 8293 & -51.56 & 1.85 & -0.85 & 66.723 \\ 8292 & -51.8 & 1.97 & -1.35 & 66.729 \\ 8291 & -52.1 & 1.89 & -1.00 & 66.736 \\ 1435 & -52.41 & 1.98 & -0.98 & 66.744\end{array}$




$\begin{array}{lrlll}8290 & -53.31 & 1.90 & -1.33 & 66.767 \\ 8289 & -53.56 & 1.85 & -0.71 & 66.773 \\ 1434 & -53.8 & 1.92 & -0.92 & 66.779 \\ 4026 & -54 & 1.92 & -1.04 & 66.784 \\ 8288 & -54.09 & 1.96 & -0.79 & 66.786 \\ 8287 & -54.25 & 1.90 & -0.44 & 66.790 \\ 8286 & -54.49 & 1.86 & -0.86 & 66.796 \\ 8285 & -54.74 & 1.90 & -0.48 & 66.802 \\ 1433 & -55 & 1.95 & -0.92 & 66.809 \\ 8284 & -55.19 & 1.88 & -0.73 & 66.814 \\ 8283 & -55.45 & 1.85 & -0.80 & 66.820 \\ 8282 & -55.7 & 1.89 & -0.79 & 66.826 \\ 8281 & -55.83 & 1.88 & -0.78 & 66.830 \\ 1432 & -55.9 & 1.97 & -0.89 & 66.831 \\ 8280 & -56.08 & 1.90 & -0.70 & 66.836 \\ 8279 & -56.32 & 2.01 & -0.86 & 66.842 \\ 1431 & -56.57 & 1.92 & -1.08 & 66.848 \\ 8278 & -56.97 & 1.92 & -0.96 & 66.858 \\ 8277 & -57.27 & 1.91 & -1.00 & 66.866 \\ 8276 & -57.52 & 1.91 & -1.12 & 66.872 \\ 1430 & -57.76 & 1.91 & -1.12 & 66.878 \\ 8275 & -57.94 & 1.96 & -1.18 & 66.882 \\ 8274 & -58.19 & 1.99 & -0.98 & 66.889 \\ 8273 & -58.44 & 2.00 & -0.98 & 66.895 \\ 1429 & -58.7 & 1.95 & -1.01 & 66.901 \\ 8272 & -59.02 & 1.93 & -1.08 & 66.909 \\ 1428 & -59.28 & 1.95 & -1.04 & 66.916 \\ 8271 & -59.5 & 2.02 & -1.15 & 66.921 \\ 8270 & -60.02 & 1.98 & -0.95 & 66.934 \\ 8269 & -60.27 & 1.86 & -0.83 & 66.941 \\ 8268 & -60.52 & 1.95 & -1.04 & 66.947 \\ 8267 & -60.71 & 1.92 & -1.21 & 66.952 \\ 1427 & -60.79 & 1.80 & -0.41 & 66.954 \\ 8266 & -60.96 & 1.87 & -1.01 & 66.958 \\ 8265 & -61.21 & 1.99 & -1.00 & 66.964 \\ 1426 & -61.42 & 1.85 & -0.86 & 66.969 \\ 8264 & -61.79 & 1.96 & -1.09 & 66.979 \\ 8263 & -62.02 & 1.85 & -0.73 & 66.984 \\ 1425 & -62.33 & 1.85 & -0.88 & 66.992 \\ 8262 & -62.63 & 1.97 & -1.12 & 67.000 \\ 1424 & -62.86 & 1.86 & -0.58 & 67.005 \\ 8261 & -63.1 & 1.92 & -1.05 & 67.011 \\ 8260 & -63.36 & 1.90 & -0.76 & 67.018 \\ 8259 & -63.61 & 1.87 & -0.89 & 67.024 \\ 1423 & -63.86 & 1.81 & -0.79 & 67.030 \\ 8258 & -64.13 & 1.86 & -1.00 & 67.037 \\ 4025 & -64.2 & 1.86 & -0.78 & 67.039 \\ 8257 & -64.32 & 1.86 & -0.95 & 67.042\end{array}$




$\begin{array}{lrlll}8256 & -64.57 & 1.99 & -1.09 & 67.048 \\ 1422 & -64.8 & 1.83 & -0.79 & 67.054 \\ 8255 & -65 & 1.89 & -0.76 & 67.059 \\ 8254 & -65.25 & 1.77 & -0.97 & 67.065 \\ 8253 & -65.51 & 1.87 & -0.80 & 67.071 \\ 1421 & -65.77 & 1.77 & -0.88 & 67.078 \\ 8252 & -66 & 1.83 & -0.86 & 67.084 \\ 8251 & -66.25 & 1.82 & -1.12 & 67.090 \\ 1420 & -66.52 & 1.81 & -0.86 & 67.097 \\ 8250 & -66.6 & 1.82 & -0.92 & 67.099 \\ 8249 & -66.85 & 1.71 & -0.97 & 67.105 \\ 8248 & -67.11 & 1.70 & -0.98 & 67.111 \\ 1419 & -67.33 & 1.74 & -0.48 & 67.117 \\ 8247 & -67.58 & 1.80 & -1.03 & 67.123 \\ 8246 & -67.83 & 1.85 & -1.10 & 67.129 \\ 8245 & -68.08 & 1.85 & -0.95 & 67.136 \\ 1418 & -68.31 & 1.73 & -1.20 & 67.141 \\ 8244 & -68.41 & 1.83 & -1.08 & 67.144 \\ 8243 & -68.66 & 1.87 & -0.72 & 67.150 \\ 8242 & -68.99 & 1.84 & -1.13 & 67.158 \\ 1417 & -69.22 & 1.82 & -0.98 & 67.164 \\ 8241 & -69.44 & 1.82 & -0.95 & 67.170 \\ 8240 & -69.69 & 1.89 & -0.90 & 67.176 \\ 8239 & -69.94 & 1.91 & -0.80 & 67.182 \\ 1416 & -70.19 & 1.91 & -0.89 & 67.188 \\ 8238 & -70.34 & 1.85 & -1.00 & 67.192 \\ 8237 & -70.59 & 1.98 & -0.87 & 67.198 \\ 8236 & -70.84 & 1.94 & -1.04 & 67.205 \\ 1415 & -71.13 & 1.86 & -1.29 & 67.212 \\ 8235 & -71.65 & 1.97 & -1.31 & 67.225 \\ 8234 & -71.9 & 1.96 & -1.14 & 67.231 \\ 8233 & -72.11 & 1.91 & -1.06 & 67.236 \\ 1414 & -72.12 & 1.87 & -0.79 & 67.237 \\ 8232 & -72.26 & 2.03 & -1.36 & 67.240 \\ 8231 & -72.5 & 2.01 & -0.99 & 67.246 \\ 8230 & -72.7 & 1.99 & -1.25 & 67.251 \\ 1413 & -72.97 & 1.92 & -0.95 & 67.258 \\ 6164 & -72.975 & 1.82 & -1.23 & 67.258 \\ 6163 & -73.05 & 1.83 & -1.00 & 67.260 \\ 6162 & -73.1 & 1.85 & -1.06 & 67.261 \\ 6161 & -73.15 & 1.86 & -1.10 & 67.262 \\ 6160 & -73.2 & 1.93 & -0.74 & 67.264 \\ 6159 & -73.25 & 1.89 & -0.91 & 67.265 \\ 6158 & -73.3 & 1.94 & -0.80 & 67.266 \\ 8229 & -73.38 & 1.97 & -1.02 & 67.268 \\ 6154 & -73.55 & 1.96 & -1.19 & 67.272 \\ 6153 & -73.595 & 2.05 & -1.03 & 67.274 \\ 8228 & -73.62 & 1.91 & -0.66 & 67.274\end{array}$




\begin{tabular}{|c|c|c|c|c|}
\hline 6152 & -73.64 & 2.03 & -0.96 & 67.275 \\
\hline 6151 & -73.69 & 2.01 & -1.08 & 67.276 \\
\hline 6149 & -73.76 & 1.91 & -1.23 & 67.278 \\
\hline 6148 & -73.81 & 1.97 & -0.97 & 67.279 \\
\hline 6147 & -73.86 & 1.94 & -0.85 & 67.280 \\
\hline 8227 & -73.87 & 1.88 & -0.72 & 67.280 \\
\hline 6146 & -73.91 & 1.86 & -0.83 & 67.281 \\
\hline 6167 & -73.96 & 1.93 & -0.80 & 67.283 \\
\hline 6145 & -74.01 & 1.88 & -0.86 & 67.284 \\
\hline 6166 & -74.06 & 1.93 & -1.00 & 67.285 \\
\hline 6144 & -74.11 & 2.03 & -1.02 & 67.286 \\
\hline 6165 & -74.16 & 2.01 & -1.12 & 67.288 \\
\hline 6143 & -74.21 & 1.98 & -0.87 & 67.289 \\
\hline 4024 & -74.3 & 1.80 & -0.95 & 67.291 \\
\hline 8226 & -74.41 & 1.93 & -0.48 & 67.294 \\
\hline 8225 & -74.66 & 1.92 & -0.78 & 67.300 \\
\hline 1411 & -75 & 1.79 & -1.00 & 67.309 \\
\hline 5628 & -76.05 & 1.86 & -0.34 & 67.335 \\
\hline 8224 & -76.12 & 1.88 & -0.77 & 67.337 \\
\hline 8223 & -76.39 & 1.86 & -0.73 & 67.343 \\
\hline 8222 & -76.64 & 1.91 & -0.84 & 67.350 \\
\hline 1410 & -76.9 & 1.75 & -0.16 & 67.356 \\
\hline 8221 & -77.21 & 1.88 & -0.80 & 67.364 \\
\hline 8220 & -77.46 & 2.02 & -0.96 & 67.370 \\
\hline 8219 & -77.71 & 2.07 & -0.73 & 67.376 \\
\hline 1409 & -77.94 & 1.84 & -0.88 & 67.382 \\
\hline 8218 & -78.07 & 2.01 & -0.69 & 67.385 \\
\hline 5627 & -78.3 & 2.15 & -0.47 & 67.391 \\
\hline 8217 & -78.32 & 2.04 & -1.11 & 67.392 \\
\hline 8216 & -78.57 & 2.04 & -0.64 & 67.398 \\
\hline 1408 & -78.87 & 1.96 & -0.63 & 67.405 \\
\hline 8215 & -79.06 & 1.96 & -1.01 & 67.410 \\
\hline 8214 & -79.31 & 2.10 & -1.23 & 67.416 \\
\hline 8213 & -79.54 & 2.14 & -1.17 & 67.422 \\
\hline 1407 & -79.8 & 2.09 & -0.68 & 67.429 \\
\hline 8212 & -79.97 & 2.12 & -1.12 & 67.433 \\
\hline 8211 & -80.22 & 2.14 & -1.01 & 67.439 \\
\hline 8210 & -80.47 & 2.04 & -0.84 & 67.445 \\
\hline 1406 & -80.71 & 2.06 & -0.53 & 67.451 \\
\hline 8209 & -80.83 & 2.02 & -0.92 & 67.454 \\
\hline 8208 & -81.08 & 2.17 & -1.09 & 67.461 \\
\hline 8207 & -81.33 & 2.01 & -0.80 & 67.467 \\
\hline 1405 & -81.58 & 1.98 & -0.97 & 67.473 \\
\hline 8206 & -81.8 & 2.06 & -0.75 & 67.479 \\
\hline 8205 & -82.05 & 2.09 & -1.01 & 67.485 \\
\hline 8204 & -82.29 & 2.04 & -1.11 & 67.491 \\
\hline 1404 & -82.57 & 2.01 & -0.83 & 67.498 \\
\hline 8203 & -82.59 & 2.13 & -0.88 & 67.498 \\
\hline
\end{tabular}




$\begin{array}{lllll}8201 & -83.06 & 2.14 & -0.77 & 67.510 \\ 1403 & -83.37 & 2.11 & -0.59 & 67.518 \\ 8200 & -83.4 & 2.24 & -0.89 & 67.519 \\ 8199 & -83.65 & 2.24 & -1.40 & 67.525 \\ 8202 & -83.83 & 2.13 & -0.98 & 67.529 \\ 8198 & -83.88 & 2.30 & -1.06 & 67.531 \\ 1402 & -84.14 & 2.27 & -0.73 & 67.537 \\ 5629 & -84.26 & 2.32 & -1.60 & 67.540 \\ 8197 & -84.34 & 2.33 & -0.97 & 67.542 \\ 8196 & -84.59 & 2.26 & -1.20 & 67.546 \\ 8195 & -84.84 & 2.27 & -1.01 & 67.551 \\ 1401 & -85.1 & 2.25 & -0.88 & 67.556 \\ 8194 & -85.29 & 2.20 & -1.13 & 67.559 \\ 8193 & -85.54 & 2.26 & -1.16 & 67.564 \\ 8192 & -85.78 & 2.24 & -1.23 & 67.569 \\ 1400 & -86.07 & 2.22 & -0.87 & 67.574 \\ 8191 & -86.28 & 2.28 & -0.99 & 67.578 \\ 8190 & -86.54 & 2.31 & -1.02 & 67.583 \\ 1399 & -86.78 & 2.26 & -0.72 & 67.587 \\ 8189 & -87.24 & 2.20 & -0.87 & 67.596 \\ 8188 & -87.49 & 2.22 & -1.65 & 67.601 \\ 8187 & -87.74 & 2.22 & -0.93 & 67.606 \\ 1398 & -87.99 & 2.19 & -1.04 & 67.610 \\ 8186 & -88.39 & 2.23 & -0.73 & 67.618 \\ 8185 & -88.63 & 2.20 & -0.88 & 67.622 \\ 8184 & -88.89 & 2.26 & -0.74 & 67.627 \\ 1397 & -89.14 & 2.23 & -0.69 & 67.632 \\ 8182 & -89.6 & 2.27 & -0.93 & 67.641 \\ 8181 & -89.84 & 2.26 & -0.70 & 67.645 \\ 1396 & -90.08 & 2.20 & -0.86 & 67.650 \\ 8180 & -90.34 & 2.38 & -1.07 & 67.655 \\ 8179 & -90.58 & 2.28 & -0.94 & 67.659 \\ 8178 & -90.83 & 2.25 & -1.05 & 67.664 \\ 8177 & -91.06 & 2.30 & -0.93 & 67.668 \\ 1395 & -91.07 & 2.25 & -0.62 & 67.668 \\ 8176 & -91.31 & 2.28 & -0.98 & 67.673 \\ 8175 & -91.56 & 2.28 & -0.91 & 67.678 \\ 1394 & -91.86 & 2.35 & -0.82 & 67.683 \\ 8174 & -92.02 & 2.31 & -0.95 & 67.686 \\ 8173 & -92.27 & 2.44 & -0.81 & 67.691 \\ 8172 & -92.52 & 2.38 & -1.10 & 67.696 \\ 1393 & -92.79 & 2.31 & -0.75 & 67.701 \\ 8171 & -92.92 & 2.23 & -1.12 & 67.703 \\ 8170 & -93.17 & 2.23 & -0.89 & 67.708 \\ 8169 & -93.42 & 2.33 & -0.74 & 67.713 \\ 1392 & -93.65 & 2.34 & -1.01 & 67.717 \\ 8168 & -93.84 & 2.27 & -1.06 & 67.720 \\ 8167 & -94.09 & 2.35 & -0.80 & 67.725\end{array}$




\begin{tabular}{|c|c|c|c|c|}
\hline 4022 & -94.15 & 2.29 & -0.94 & 67.726 \\
\hline 1391 & -94.32 & 2.29 & -1.03 & 67.730 \\
\hline 8166 & -94.74 & 2.28 & -0.95 & 67.737 \\
\hline 8165 & -94.99 & 2.20 & -0.89 & 67.742 \\
\hline 5626 & -95.1 & 2.17 & -1.14 & 67.744 \\
\hline 8164 & -95.24 & 2.20 & -0.86 & 67.747 \\
\hline 1390 & -95.53 & 2.14 & -1.02 & 67.752 \\
\hline 8163 & -95.68 & 2.31 & -0.78 & 67.755 \\
\hline 8162 & -95.94 & 2.28 & -1.00 & 67.760 \\
\hline 8161 & -96.19 & 2.32 & -0.65 & 67.765 \\
\hline 1389 & -96.46 & 2.27 & -0.88 & 67.770 \\
\hline 8160 & -96.59 & 2.28 & -1.04 & 67.772 \\
\hline 8159 & -96.84 & 2.28 & -1.07 & 67.777 \\
\hline 8158 & -97.09 & 2.30 & -1.20 & 67.782 \\
\hline 1388 & -97.32 & 2.22 & -1.16 & 67.786 \\
\hline 8157 & -97.69 & 2.26 & -1.13 & 67.793 \\
\hline 8156 & -97.94 & 2.30 & -0.94 & 67.798 \\
\hline 1387 & -98.16 & 2.28 & -0.99 & 67.802 \\
\hline 8155 & -99.05 & 2.34 & -0.87 & 67.819 \\
\hline 8154 & -99.28 & 2.26 & -1.02 & 67.823 \\
\hline 1386 & -99.43 & 2.40 & -0.83 & 67.826 \\
\hline 8153 & -99.55 & 2.25 & -1.18 & 67.828 \\
\hline 8152 & -99.83 & 2.30 & -0.65 & 67.833 \\
\hline 8151 & -100.19 & 2.16 & -1.07 & 67.840 \\
\hline 1385 & -100.32 & 2.26 & -1.00 & 67.843 \\
\hline 8150 & -100.71 & 2.21 & -0.99 & 67.850 \\
\hline 8149 & -100.96 & 2.33 & -0.59 & 67.855 \\
\hline 1384 & -101.25 & 2.26 & -1.03 & 67.860 \\
\hline 8148 & -101.49 & 2.26 & -1.08 & 67.865 \\
\hline 8147 & -101.74 & 2.29 & -1.40 & 67.869 \\
\hline 8146 & -101.99 & 2.29 & -0.96 & 67.874 \\
\hline 5270 & -102.12 & 2.29 & -1.02 & 67.876 \\
\hline 1383 & -102.15 & 2.34 & -1.08 & 67.877 \\
\hline 5269 & -102.17 & 2.27 & -1.06 & 67.877 \\
\hline 5268 & -102.22 & 2.27 & -1.11 & 67.878 \\
\hline 5267 & -102.27 & 2.23 & -1.16 & 67.879 \\
\hline 8145 & -102.34 & 2.30 & -1.15 & 67.881 \\
\hline 5266 & -102.37 & 2.24 & -1.06 & 67.881 \\
\hline 5265 & -102.41 & 2.26 & -1.20 & 67.882 \\
\hline 5264 & -102.46 & 2.25 & -0.98 & 67.883 \\
\hline 8144 & -102.5 & 2.30 & -0.95 & 67.884 \\
\hline 5263 & -102.51 & 2.22 & -0.88 & 67.884 \\
\hline 5262 & -102.6 & 2.30 & -0.91 & 67.886 \\
\hline 5261 & -102.65 & 2.27 & -1.03 & 67.886 \\
\hline 5260 & -102.7 & 2.23 & -0.68 & 67.887 \\
\hline 5259 & -102.75 & 2.31 & -0.96 & 67.888 \\
\hline 5258 & -102.81 & 2.27 & -0.98 & 67.889 \\
\hline 5257 & -102.84 & 2.27 & -1.19 & 67.890 \\
\hline
\end{tabular}




\begin{tabular}{|c|c|c|c|c|}
\hline 5622 & -102.86 & 2.30 & -1.18 & 67.890 \\
\hline 5255 & -102.91 & 2.24 & -1.08 & 67.891 \\
\hline 5254 & -102.95 & 2.20 & -0.68 & 67.892 \\
\hline 1382 & -103.02 & 2.30 & -0.94 & 67.893 \\
\hline 5252 & -103.07 & 2.20 & -0.87 & 67.894 \\
\hline 5251 & -103.11 & 2.26 & -1.21 & 67.895 \\
\hline 5250 & -103.16 & 2.37 & -0.75 & 67.896 \\
\hline 8143 & -103.2 & 2.33 & -1.15 & 67.897 \\
\hline 5249 & -103.26 & 2.23 & -0.92 & 67.898 \\
\hline 5248 & -103.28 & 2.30 & -0.71 & 67.898 \\
\hline 5246 & -103.35 & 2.20 & -0.65 & 67.900 \\
\hline 5245 & -103.36 & 2.24 & -0.97 & 67.900 \\
\hline 5244 & -103.41 & 2.29 & -0.93 & 67.901 \\
\hline 8142 & -103.45 & 2.34 & -1.13 & 67.902 \\
\hline 5243 & -103.51 & 2.14 & -0.96 & 67.903 \\
\hline 5242 & -103.53 & 2.20 & -0.94 & 67.903 \\
\hline 5241 & -103.55 & 2.14 & -0.72 & 67.903 \\
\hline 5240 & -103.57 & 2.28 & -1.05 & 67.904 \\
\hline 5239 & -103.59 & 2.28 & -0.93 & 67.904 \\
\hline 5238 & -103.62 & 2.19 & -0.95 & 67.905 \\
\hline 5237 & -103.65 & 2.34 & -1.12 & 67.905 \\
\hline 5236 & -103.67 & 2.23 & -1.19 & 67.906 \\
\hline 5235 & -103.72 & 2.29 & -1.24 & 67.907 \\
\hline 8141 & -103.76 & 2.33 & -1.17 & 67.907 \\
\hline 5234 & -103.82 & 2.26 & -0.91 & 67.909 \\
\hline 5233 & -103.87 & 2.32 & -0.96 & 67.909 \\
\hline 5232 & -103.92 & 2.30 & -0.96 & 67.910 \\
\hline 5231 & -103.97 & 2.32 & -1.04 & 67.911 \\
\hline 1381 & -104 & 2.26 & -0.81 & 67.912 \\
\hline 4021 & -104.08 & 2.11 & -1.02 & 67.913 \\
\hline 5230 & -104.08 & 2.32 & -1.10 & 67.913 \\
\hline 5229 & -104.12 & 2.37 & -0.93 & 67.914 \\
\hline 8140 & -104.16 & 2.31 & -0.92 & 67.915 \\
\hline 5227 & -104.27 & 2.28 & -1.04 & 67.917 \\
\hline 5226 & -104.31 & 2.37 & -0.93 & 67.918 \\
\hline 5225 & -104.38 & 2.32 & -0.68 & 67.919 \\
\hline 8139 & -104.43 & 2.23 & -1.19 & 67.920 \\
\hline 5224 & -104.47 & 2.32 & -1.17 & 67.921 \\
\hline 5205 & -104.49 & 2.27 & -0.91 & 67.921 \\
\hline 5223 & -104.52 & 2.35 & -1.03 & 67.922 \\
\hline 5222 & -104.57 & 2.25 & -1.10 & 67.923 \\
\hline 5204 & -104.59 & 2.28 & -0.78 & 67.923 \\
\hline 5221 & -104.62 & 2.29 & -1.15 & 67.924 \\
\hline 5203 & -104.64 & 2.22 & -0.85 & 67.924 \\
\hline 8138 & -104.66 & 2.26 & -1.10 & 67.924 \\
\hline 5202 & -104.69 & 2.13 & -0.90 & 67.925 \\
\hline 5220 & -104.72 & 2.27 & -1.04 & 67.925 \\
\hline 5201 & -104.74 & 2.21 & -0.84 & 67.926 \\
\hline
\end{tabular}




$\begin{array}{lllll}5219 & -104.77 & 2.23 & -1.11 & 67.926 \\ 5200 & -104.79 & 2.29 & -0.89 & 67.927 \\ 5218 & -104.82 & 2.32 & -0.97 & 67.927 \\ 1380 & -104.91 & 2.36 & -0.90 & 67.929 \\ 5216 & -104.98 & 2.27 & -1.00 & 67.930 \\ 8137 & -105.03 & 2.35 & -1.62 & 67.931 \\ 5211 & -105.16 & 2.24 & -0.87 & 67.934 \\ 5215 & -105.18 & 2.22 & -0.94 & 67.934 \\ 5210 & -105.24 & 2.16 & -0.85 & 67.935 \\ 8136 & -105.27 & 2.27 & -0.81 & 67.936 \\ 5209 & -105.3 & 2.33 & -1.42 & 67.936 \\ 5208 & -105.34 & 2.31 & -0.92 & 67.937 \\ 5207 & -105.39 & 2.41 & -0.96 & 67.938 \\ 5206 & -105.44 & 2.33 & -0.81 & 67.939 \\ 8135 & -105.53 & 2.30 & -0.77 & 67.941 \\ 1379 & -105.81 & 2.13 & -0.84 & 67.946 \\ 8134 & -106.03 & 2.33 & -1.19 & 67.950 \\ 8133 & -106.28 & 2.39 & -1.14 & 67.955 \\ 5620 & -106.37 & 2.47 & -0.67 & 67.957 \\ 8132 & -106.54 & 2.28 & -0.96 & 67.960 \\ 1378 & -106.79 & 2.24 & -1.01 & 67.964 \\ 8131 & -106.92 & 2.20 & -1.01 & 67.967 \\ 5228 & -107.22 & 2.24 & -0.84 & 67.973 \\ 8029 & -107.4 & 2.25 & -1.97 & 67.976 \\ 1377 & -107.6 & 2.31 & -0.86 & 67.980 \\ 8028 & -107.89 & 2.32 & -1.09 & 67.985 \\ 8027 & -108.14 & 2.27 & -1.40 & 67.990 \\ 8026 & -108.4 & 2.31 & -1.10 & 67.995 \\ 1376 & -108.65 & 2.54 & -0.75 & 67.999 \\ 8025 & -108.86 & 2.48 & -0.92 & 68.003 \\ 8024 & -109.12 & 2.35 & -1.08 & 68.008 \\ 8023 & -109.37 & 2.26 & -1.18 & 68.013 \\ 1375 & -109.62 & 2.10 & -0.78 & 68.018 \\ 8022 & -110.11 & 2.27 & -0.95 & 68.027 \\ 8021 & -110.36 & 2.27 & -0.92 & 68.032 \\ 1374 & -110.58 & 2.18 & -1.29 & 68.036 \\ 8020 & -110.94 & 2.32 & -1.52 & 68.043 \\ 8019 & -111.16 & 2.30 & -1.26 & 68.047 \\ 8018 & -111.41 & 2.20 & -1.03 & 68.051 \\ 1373 & -111.64 & 2.28 & -0.91 & 68.056 \\ 8017 & -111.85 & 2.27 & -1.06 & 68.060 \\ 8016 & -112.05 & 2.29 & -1.19 & 68.064 \\ 8015 & -112.3 & 2.29 & -1.15 & 68.068 \\ 1372 & -112.54 & 2.15 & -1.04 & 68.073 \\ 8014 & -112.87 & 2.25 & -1.08 & 68.079 \\ 8013 & -113.12 & 2.26 & -1.11 & 68.083 \\ 1371 & -113.37 & 2.23 & -1.15 & 68.087 \\ 8012 & -114.09 & 2.54 & -0.84 & 68.097\end{array}$




\begin{tabular}{|c|c|c|c|c|}
\hline 4020 & -114.3 & 2.19 & -0.82 & 68.100 \\
\hline 8011 & -114.32 & 2.38 & -1.04 & 68.101 \\
\hline 8010 & -114.58 & 2.32 & -1.14 & 68.105 \\
\hline 1370 & -114.81 & 2.25 & -1.14 & 68.108 \\
\hline 8009 & -115.12 & 2.43 & -0.73 & 68.113 \\
\hline 8008 & -115.37 & 2.48 & -0.89 & 68.117 \\
\hline 1369 & -115.66 & 2.44 & -1.16 & 68.121 \\
\hline 8007 & -115.8 & 2.39 & -1.18 & 68.123 \\
\hline 8006 & -116.05 & 2.46 & -0.76 & 68.127 \\
\hline 8005 & -116.3 & 2.53 & -0.87 & 68.131 \\
\hline 1368 & -116.6 & 2.35 & -0.93 & 68.135 \\
\hline 8004 & -116.77 & 2.52 & -1.48 & 68.138 \\
\hline 8003 & -117.02 & 2.44 & -1.04 & 68.141 \\
\hline 8002 & -117.28 & 2.56 & -0.63 & 68.145 \\
\hline 1367 & -117.54 & 2.30 & -0.78 & 68.149 \\
\hline 8001 & -117.65 & 2.35 & -1.07 & 68.151 \\
\hline 8000 & -118.03 & 2.26 & -1.09 & 68.157 \\
\hline 7999 & -118.28 & 2.39 & -0.75 & 68.160 \\
\hline 1366 & -118.55 & 2.43 & -0.98 & 68.164 \\
\hline 7998 & -118.64 & 2.46 & -0.88 & 68.166 \\
\hline 7997 & -118.89 & 2.40 & -0.81 & 68.170 \\
\hline 7996 & -119.16 & 2.37 & -1.50 & 68.174 \\
\hline 1365 & -119.41 & 2.34 & -0.95 & 68.177 \\
\hline 7995 & -119.54 & 2.40 & -0.99 & 68.179 \\
\hline 7994 & -119.78 & 2.40 & -0.95 & 68.183 \\
\hline 7993 & -120.02 & 2.46 & -1.11 & 68.186 \\
\hline 1364 & -120.35 & 2.39 & -0.98 & 68.191 \\
\hline 7992 & -120.46 & 2.32 & -1.13 & 68.193 \\
\hline 7991 & -120.71 & 2.35 & -0.47 & 68.197 \\
\hline 7990 & -120.96 & 2.42 & -0.91 & 68.201 \\
\hline 1363 & -121.25 & 2.36 & -1.03 & 68.205 \\
\hline 7989 & -121.55 & 2.41 & -1.04 & 68.209 \\
\hline 7989 & -121.55 & 2.43 & -1.00 & 68.209 \\
\hline 7988 & -121.8 & 2.39 & -0.79 & 68.213 \\
\hline 7987 & -122.05 & 2.41 & -0.75 & 68.217 \\
\hline 1362 & -122.28 & 2.43 & -1.07 & 68.220 \\
\hline 7986 & -122.64 & 2.42 & -0.72 & 68.226 \\
\hline 7985 & -122.89 & 2.33 & -0.94 & 68.230 \\
\hline 1361 & -123.1 & 2.27 & -1.18 & 68.233 \\
\hline 7984 & -123.36 & 2.40 & -0.87 & 68.237 \\
\hline 7983 & -123.61 & 2.31 & -0.90 & 68.240 \\
\hline 1360 & -123.82 & 2.33 & -1.13 & 68.244 \\
\hline 7982 & -124.29 & 2.37 & -0.69 & 68.251 \\
\hline 4019 & -124.5 & 2.31 & -1.11 & 68.254 \\
\hline 7981 & -124.53 & 2.38 & -0.87 & 68.254 \\
\hline 7980 & -124.78 & 2.42 & -0.82 & 68.258 \\
\hline 1359 & -125.01 & 2.25 & -0.91 & 68.262 \\
\hline 7979 & -125.28 & 2.35 & -0.94 & 68.266 \\
\hline
\end{tabular}




\begin{tabular}{|c|c|c|c|c|}
\hline 7978 & -125.53 & 2.27 & -0.56 & 68.269 \\
\hline 1358 & -125.85 & 2.18 & -0.90 & 68.274 \\
\hline 7977 & -126.17 & 2.37 & -1.19 & 68.279 \\
\hline 7976 & -126.42 & 2.43 & -0.81 & 68.283 \\
\hline 7975 & -126.67 & 2.41 & -0.73 & 68.286 \\
\hline 1357 & -126.9 & 2.28 & -1.17 & 68.290 \\
\hline 7974 & -127.13 & 2.42 & -0.72 & 68.293 \\
\hline 7973 & -127.38 & 2.34 & -0.78 & 68.297 \\
\hline 7972 & -127.63 & 2.46 & -0.78 & 68.301 \\
\hline 1356 & -127.85 & 2.38 & -0.73 & 68.304 \\
\hline 7971 & -128.15 & 2.50 & -0.86 & 68.309 \\
\hline 7970 & -128.38 & 2.43 & -1.50 & 68.312 \\
\hline 7969 & -128.64 & 2.47 & -0.85 & 68.316 \\
\hline 1355 & -128.84 & 2.34 & -0.84 & 68.319 \\
\hline 7968 & -129.12 & 2.41 & -0.89 & 68.323 \\
\hline 7967 & -129.35 & 2.42 & -1.04 & 68.327 \\
\hline 7966 & -129.6 & 2.45 & -0.92 & 68.331 \\
\hline 1354 & -129.91 & 2.44 & -0.76 & 68.335 \\
\hline 7965 & -129.95 & 2.45 & -0.94 & 68.336 \\
\hline 7964 & -130.2 & 2.45 & -1.12 & 68.340 \\
\hline 7963 & -130.45 & 2.47 & -1.09 & 68.343 \\
\hline 1353 & -130.73 & 2.42 & -1.00 & 68.348 \\
\hline 7962 & -130.96 & 2.54 & -0.98 & 68.351 \\
\hline 7961 & -131.21 & 2.53 & -0.93 & 68.355 \\
\hline 7960 & -131.46 & 2.50 & -0.86 & 68.358 \\
\hline 1352 & -131.71 & 2.38 & -1.10 & 68.362 \\
\hline 7959 & -132 & 2.45 & -0.98 & 68.367 \\
\hline 7958 & -132.29 & 2.44 & -0.87 & 68.371 \\
\hline 7957 & -132.45 & 2.25 & -1.17 & 68.373 \\
\hline 1351 & -132.53 & 2.38 & -0.92 & 68.375 \\
\hline 7956 & -132.82 & 2.46 & -0.95 & 68.379 \\
\hline 7955 & -133.08 & 2.48 & -0.97 & 68.383 \\
\hline 1350 & -133.3 & 2.43 & -0.68 & 68.386 \\
\hline 5618 & -133.49 & 2.39 & -0.46 & 68.389 \\
\hline 7954 & -133.56 & 2.47 & -1.29 & 68.390 \\
\hline 7953 & -133.68 & 2.41 & -0.98 & 68.392 \\
\hline 7952 & -133.93 & 2.31 & -0.97 & 68.396 \\
\hline 4018 & -134 & 2.42 & -0.94 & 68.397 \\
\hline 1349 & -134.2 & 2.36 & -0.98 & 68.400 \\
\hline 7951 & -134.34 & 2.31 & -1.08 & 68.402 \\
\hline 7950 & -134.62 & 2.36 & -1.38 & 68.406 \\
\hline 7949 & -134.88 & 2.50 & -1.00 & 68.410 \\
\hline 1348 & -135.13 & 2.47 & -0.92 & 68.414 \\
\hline 7948 & -135.38 & 2.41 & -1.24 & 68.417 \\
\hline 7947 & -135.63 & 2.48 & -1.12 & 68.421 \\
\hline 1347 & -135.86 & 2.44 & -1.00 & 68.425 \\
\hline 7946 & -136.27 & 2.40 & -1.34 & 68.431 \\
\hline 7945 & -136.54 & 2.29 & -1.03 & 68.435 \\
\hline
\end{tabular}




\begin{tabular}{|c|c|c|c|c|}
\hline 7944 & -136.76 & 2.34 & -0.78 & 68.438 \\
\hline 1346 & -137.14 & 2.29 & -0.80 & 68.444 \\
\hline 7943 & -137.18 & 2.30 & -0.67 & 68.444 \\
\hline 7942 & -137.47 & 2.46 & -0.93 & 68.449 \\
\hline 7941 & -137.68 & 2.48 & -0.71 & 68.452 \\
\hline 1345 & -138.08 & 2.44 & -1.16 & 68.458 \\
\hline 7940 & -138.16 & 2.54 & -1.02 & 68.459 \\
\hline 7939 & -138.52 & 2.38 & -1.59 & 68.465 \\
\hline 1344 & -138.79 & 2.46 & -0.83 & 68.469 \\
\hline 7938 & -138.85 & 2.42 & -1.88 & 68.470 \\
\hline 7937 & -139.09 & 2.48 & -1.08 & 68.473 \\
\hline 7936 & -139.36 & 2.33 & -1.11 & 68.477 \\
\hline 1343 & -139.74 & 2.46 & -1.06 & 68.483 \\
\hline 7935 & -139.99 & 2.40 & -0.90 & 68.487 \\
\hline 7934 & -140.24 & 2.48 & -0.88 & 68.490 \\
\hline 7933 & -140.49 & 2.51 & -1.24 & 68.494 \\
\hline 1342 & -140.64 & 2.52 & -1.04 & 68.497 \\
\hline 7932 & -140.82 & 2.40 & -1.22 & 68.499 \\
\hline 7931 & -141.07 & 2.34 & -1.15 & 68.503 \\
\hline 7930 & -141.32 & 2.41 & -1.09 & 68.507 \\
\hline 1341 & -141.52 & 2.40 & -1.28 & 68.510 \\
\hline 7929 & -141.77 & 2.41 & -1.16 & 68.513 \\
\hline 7928 & -142.11 & 2.39 & -1.24 & 68.519 \\
\hline 7927 & -142.36 & 2.43 & -1.15 & 68.522 \\
\hline 1340 & -142.59 & 2.37 & -0.78 & 68.526 \\
\hline 7926 & -142.83 & 2.38 & -1.21 & 68.529 \\
\hline 7925 & -143.08 & 2.46 & -1.04 & 68.533 \\
\hline 7924 & -143.33 & 2.36 & -1.07 & 68.537 \\
\hline 1339 & -143.39 & 2.51 & -0.84 & 68.538 \\
\hline 7923 & -143.46 & 2.37 & -1.11 & 68.539 \\
\hline 7922 & -143.71 & 2.40 & -1.21 & 68.543 \\
\hline 4017 & -143.95 & 2.40 & -0.99 & 68.546 \\
\hline 7921 & -143.96 & 2.37 & -1.08 & 68.546 \\
\hline 1338 & -144.32 & 2.43 & -1.24 & 68.552 \\
\hline 7920 & -144.52 & 2.39 & -1.23 & 68.555 \\
\hline 7919 & -144.77 & 2.42 & -1.04 & 68.559 \\
\hline 7918 & -145.02 & 2.44 & -1.20 & 68.562 \\
\hline 1337 & -145.25 & 2.46 & -1.27 & 68.566 \\
\hline 7917 & -145.5 & 2.35 & -1.30 & 68.570 \\
\hline 7916 & -145.73 & 2.41 & -1.05 & 68.573 \\
\hline 7915 & -145.98 & 2.44 & -1.04 & 68.577 \\
\hline 1336 & -146.22 & 2.38 & -1.34 & 68.580 \\
\hline 7914 & -146.38 & 2.42 & -1.21 & 68.583 \\
\hline 7913 & -146.63 & 2.48 & -1.19 & 68.587 \\
\hline 7912 & -146.88 & 2.40 & -1.22 & 68.590 \\
\hline 1335 & -147.2 & 2.38 & -1.04 & 68.595 \\
\hline 7911 & -147.34 & 2.41 & -1.24 & 68.597 \\
\hline 7910 & -147.59 & 2.42 & -1.22 & 68.601 \\
\hline
\end{tabular}




$\begin{array}{lrlll}7909 & -147.84 & 2.42 & -1.00 & 68.605 \\ 7908 & -148.38 & 2.42 & -1.22 & 68.613 \\ 7907 & -148.63 & 2.33 & -1.23 & 68.617 \\ 1334 & -148.73 & 2.49 & -1.04 & 68.618 \\ 7906 & -148.88 & 2.45 & -1.07 & 68.620 \\ 1333 & -149.08 & 2.43 & -1.24 & 68.623 \\ 7905 & -149.18 & 2.37 & -1.30 & 68.625 \\ 7904 & -149.43 & 2.42 & -1.21 & 68.629 \\ 7903 & -149.68 & 2.44 & -1.31 & 68.632 \\ 1332 & -149.94 & 2.33 & -1.24 & 68.636 \\ 7902 & -150.07 & 2.35 & -1.16 & 68.638 \\ 7901 & -150.32 & 2.39 & -1.10 & 68.642 \\ 7900 & -150.57 & 2.32 & -1.89 & 68.646 \\ 1331 & -150.85 & 2.39 & -1.21 & 68.650 \\ 7899 & -151.1 & 2.51 & -1.23 & 68.654 \\ 7898 & -151.35 & 2.46 & -1.07 & 68.658 \\ 7897 & -151.6 & 2.43 & -1.32 & 68.661 \\ 1330 & -151.89 & 2.32 & -1.45 & 68.666 \\ 7896 & -152.11 & 2.39 & -1.33 & 68.669 \\ 7895 & -152.36 & 2.40 & -1.23 & 68.673 \\ 7894 & -152.61 & 2.41 & -1.27 & 68.676 \\ 1329 & -152.83 & 2.46 & -0.99 & 68.680 \\ 7893 & -152.95 & 2.47 & -1.04 & 68.682 \\ 7892 & -153.2 & 2.35 & -1.27 & 68.685 \\ 7891 & -153.45 & 2.58 & -1.38 & 68.689 \\ 1328 & -153.68 & 2.51 & -1.29 & 68.693 \\ 7890 & -153.82 & 2.62 & -1.30 & 68.695 \\ 7889 & -154.1 & 2.59 & -1.15 & 68.699 \\ 7888 & -154.37 & 2.59 & -1.22 & 68.703 \\ 1327 & -154.55 & 2.59 & -1.37 & 68.706 \\ 4016 & -154.6 & 2.50 & -1.06 & 68.706 \\ 7887 & -155.08 & 2.55 & -1.25 & 68.714 \\ 7886 & -155.33 & 2.51 & -1.20 & 68.717 \\ 7885 & -155.58 & 2.58 & -0.99 & 68.721 \\ 1326 & -155.6 & 2.49 & -0.94 & 68.721 \\ 7780 & -155.82 & 2.47 & -1.34 & 68.725 \\ 7884 & -155.93 & 2.48 & -1.15 & 68.726 \\ 7883 & -156.18 & 2.59 & -1.15 & 68.730 \\ 1325 & -156.4 & 2.54 & -0.95 & 68.733 \\ 7882 & -156.42 & 2.58 & -0.75 & 68.734 \\ 7881 & -156.79 & 2.48 & -1.07 & 68.739 \\ 7880 & -157.04 & 2.43 & -0.89 & 68.743 \\ 7879 & -157.29 & 2.46 & -1.30 & 68.747 \\ 1324 & -157.37 & 2.59 & -1.07 & 68.748 \\ 7878 & -157.87 & 2.47 & -1.42 & 68.756 \\ 5617 & -157.95 & 2.44 & -0.85 & 68.757 \\ 7777 & -158.1 & 2.44 & -1.37 & 68.759 \\ 7877 & -158.12 & 2.60 & -1.22 & 68.759\end{array}$




\begin{tabular}{|c|c|c|c|c|}
\hline 1323 & -158.35 & 2.41 & -1.19 & 68.763 \\
\hline 7876 & -158.37 & 2.49 & -1.64 & 68.763 \\
\hline 7775 & -158.42 & 2.48 & -1.38 & 68.764 \\
\hline 7776 & -158.69 & 2.51 & -1.60 & 68.768 \\
\hline 7778 & -159.24 & 2.54 & -1.26 & 68.776 \\
\hline 1322 & -159.32 & 2.44 & -0.79 & 68.777 \\
\hline 7779 & -159.55 & 2.42 & -1.16 & 68.781 \\
\hline 7875 & -160.07 & 2.34 & -1.26 & 68.789 \\
\hline 1321 & -160.26 & 2.44 & -1.12 & 68.791 \\
\hline 7874 & -160.39 & 2.37 & -1.26 & 68.793 \\
\hline 7873 & -160.64 & 2.41 & -1.27 & 68.797 \\
\hline 7872 & -160.89 & 2.27 & -1.24 & 68.801 \\
\hline 1320 & -161.03 & 2.36 & -0.99 & 68.803 \\
\hline 7871 & -161.25 & 2.34 & -1.01 & 68.806 \\
\hline 7870 & -161.5 & 2.37 & -1.64 & 68.810 \\
\hline 7869 & -161.75 & 2.40 & -1.11 & 68.814 \\
\hline 1319 & -161.92 & 2.40 & -0.97 & 68.816 \\
\hline 7868 & -162.22 & 2.56 & -1.00 & 68.821 \\
\hline 7867 & -162.47 & 2.43 & -1.06 & 68.825 \\
\hline 7866 & -162.72 & 2.54 & -1.12 & 68.828 \\
\hline 1318 & -162.95 & 2.37 & -1.13 & 68.832 \\
\hline 7865 & -163.19 & 2.34 & -1.18 & 68.836 \\
\hline 7864 & -163.44 & 2.38 & -1.11 & 68.839 \\
\hline 7863 & -163.69 & 2.39 & -1.29 & 68.843 \\
\hline 4015 & -164 & 2.51 & -1.21 & 68.848 \\
\hline 7861 & -164.33 & 2.51 & -1.25 & 68.853 \\
\hline 7860 & -164.58 & 2.50 & -1.18 & 68.856 \\
\hline 1316 & -164.8 & 2.41 & -1.18 & 68.860 \\
\hline 7859 & -165.14 & 2.42 & -1.05 & 68.865 \\
\hline 7858 & -165.39 & 2.42 & -1.15 & 68.869 \\
\hline 7857 & -165.63 & 2.43 & -1.13 & 68.872 \\
\hline 1315 & -165.84 & 2.48 & -1.22 & 68.875 \\
\hline 7856 & -166.01 & 2.53 & -1.10 & 68.878 \\
\hline 7855 & -166.26 & 2.49 & -1.11 & 68.882 \\
\hline 7854 & -166.51 & 2.46 & -1.07 & 68.885 \\
\hline 1314 & -166.78 & 2.43 & -1.30 & 68.889 \\
\hline 7853 & -166.97 & 2.42 & -1.13 & 68.892 \\
\hline 7852 & -167.21 & 2.37 & -1.31 & 68.896 \\
\hline 7851 & -167.45 & 2.45 & -1.06 & 68.900 \\
\hline 1313 & -167.69 & 2.25 & -1.10 & 68.903 \\
\hline 7850 & -167.84 & 2.41 & -1.22 & 68.905 \\
\hline 7849 & -168.09 & 2.40 & -1.14 & 68.909 \\
\hline 7848 & -168.34 & 2.40 & -1.17 & 68.913 \\
\hline 1312 & -168.57 & 2.31 & -0.57 & 68.916 \\
\hline 7847 & -168.81 & 2.18 & -1.27 & 68.920 \\
\hline 7846 & -169.06 & 2.25 & -1.09 & 68.924 \\
\hline 7845 & -169.31 & 2.27 & -0.86 & 68.928 \\
\hline 1311 & -169.51 & 2.23 & -0.40 & 68.931 \\
\hline
\end{tabular}




\begin{tabular}{|c|c|c|c|c|}
\hline 7844 & -169.69 & 2.33 & -0.62 & 68.933 \\
\hline 7843 & -169.94 & 2.27 & -1.35 & 68.937 \\
\hline 7842 & -170.18 & 2.35 & -1.27 & 68.941 \\
\hline 1310 & -170.5 & 2.49 & -1.16 & 68.945 \\
\hline 7841 & -170.6 & 2.44 & -1.28 & 68.947 \\
\hline 7840 & -170.85 & 2.40 & -1.28 & 68.951 \\
\hline 7839 & -171.09 & 2.50 & -1.36 & 68.954 \\
\hline 1309 & -171.44 & 2.53 & -0.98 & 68.960 \\
\hline 7838 & -171.49 & 2.66 & -1.14 & 68.960 \\
\hline 7837 & -171.74 & 2.71 & -1.11 & 68.964 \\
\hline 7836 & -172 & 2.63 & -1.01 & 68.968 \\
\hline 1308 & -172.25 & 2.60 & -1.15 & 68.972 \\
\hline 7835 & -172.42 & 2.52 & -1.17 & 68.974 \\
\hline 7834 & -172.68 & 2.51 & -1.03 & 68.978 \\
\hline 7833 & -172.92 & 2.55 & -0.97 & 68.982 \\
\hline 1307 & -173.29 & 2.47 & -1.04 & 68.987 \\
\hline 7832 & -173.48 & 2.46 & -0.93 & 68.990 \\
\hline 7831 & -173.71 & 2.52 & -1.06 & 68.994 \\
\hline 7830 & -173.94 & 2.58 & -1.31 & 68.997 \\
\hline 4014 & -174.15 & 2.51 & -1.23 & 69.000 \\
\hline 1306 & -174.24 & 2.58 & -1.04 & 69.002 \\
\hline 7829 & -174.44 & 2.59 & -1.20 & 69.005 \\
\hline 5616 & -174.63 & 2.49 & -0.64 & 69.008 \\
\hline 7828 & -174.69 & 2.51 & -1.22 & 69.008 \\
\hline 7827 & -174.93 & 2.52 & -0.98 & 69.012 \\
\hline 1305 & -175.22 & 2.40 & -0.93 & 69.016 \\
\hline 7826 & -175.33 & 2.54 & -1.33 & 69.018 \\
\hline 7825 & -175.56 & 2.53 & -1.18 & 69.022 \\
\hline 7824 & -175.81 & 2.37 & -1.20 & 69.025 \\
\hline 1304 & -175.99 & 2.44 & -1.23 & 69.028 \\
\hline 7823 & -176.26 & 2.36 & -1.30 & 69.032 \\
\hline 7822 & -176.51 & 2.37 & -0.99 & 69.036 \\
\hline 7821 & -176.76 & 2.45 & -1.26 & 69.040 \\
\hline 1303 & -176.94 & 2.43 & -1.20 & 69.042 \\
\hline 7820 & -177.03 & 2.45 & -1.27 & 69.044 \\
\hline 7819 & -177.28 & 2.52 & -1.21 & 69.047 \\
\hline 7818 & -177.53 & 2.41 & -1.14 & 69.051 \\
\hline 1302 & -177.77 & 2.52 & -1.39 & 69.055 \\
\hline 7817 & -178.08 & 2.42 & -1.07 & 69.059 \\
\hline 7816 & -178.33 & 2.41 & -1.07 & 69.063 \\
\hline 7815 & -178.58 & 2.50 & -1.02 & 69.067 \\
\hline 1301 & -178.87 & 2.67 & -1.23 & 69.071 \\
\hline 7814 & -178.98 & 2.49 & -1.13 & 69.073 \\
\hline 7813 & -179.23 & 2.54 & -1.00 & 69.077 \\
\hline 7812 & -179.48 & 2.47 & -1.13 & 69.080 \\
\hline 1300 & -179.79 & 2.48 & -1.22 & 69.085 \\
\hline 7811 & -179.88 & 2.44 & -1.24 & 69.086 \\
\hline 7810 & -180.15 & 2.62 & -1.10 & 69.091 \\
\hline
\end{tabular}




\begin{tabular}{|c|c|c|c|c|}
\hline 7809 & -180.4 & 2.61 & -1.17 & 69.094 \\
\hline 1299 & -180.7 & 2.51 & -1.39 & 69.099 \\
\hline 7808 & -180.85 & 2.59 & -1.06 & 69.101 \\
\hline 7807 & -181.11 & 2.56 & -1.54 & 69.105 \\
\hline 7806 & -181.35 & 2.51 & -1.62 & 69.109 \\
\hline 1298 & -181.68 & 2.38 & -1.18 & 69.114 \\
\hline 7805 & -181.78 & 2.42 & -1.39 & 69.115 \\
\hline 7804 & -182.02 & 2.45 & -1.58 & 69.119 \\
\hline 7803 & -182.33 & 2.41 & -1.45 & 69.123 \\
\hline 1297 & -182.58 & 2.47 & -1.13 & 69.127 \\
\hline 7802 & -182.77 & 2.47 & -1.47 & 69.130 \\
\hline 7801 & -183.01 & 2.52 & -1.48 & 69.134 \\
\hline 7800 & -183.26 & 2.51 & -1.49 & 69.137 \\
\hline 7799 & -183.37 & 2.44 & -1.12 & 69.139 \\
\hline 1296 & -183.51 & 2.51 & -1.22 & 69.141 \\
\hline 7798 & -183.61 & 2.46 & -1.26 & 69.143 \\
\hline 7797 & -183.77 & 2.40 & -1.52 & 69.145 \\
\hline 1295 & -184.1 & 2.54 & -1.62 & 69.150 \\
\hline 4013 & -184.15 & 2.59 & -1.53 & 69.151 \\
\hline 7796 & -184.47 & 2.48 & -0.92 & 69.155 \\
\hline 7795 & -184.7 & 2.49 & -1.32 & 69.159 \\
\hline 1294 & -185.1 & 2.57 & -1.16 & 69.165 \\
\hline 7794 & -185.22 & 2.53 & -0.96 & 69.167 \\
\hline 7793 & -185.48 & 2.48 & -1.28 & 69.171 \\
\hline 1293 & -185.85 & 2.56 & -1.25 & 69.176 \\
\hline 7792 & -185.97 & 2.57 & -1.18 & 69.178 \\
\hline 7791 & -186.23 & 2.55 & -1.04 & 69.182 \\
\hline 7790 & -186.5 & 2.59 & -1.24 & 69.186 \\
\hline 1292 & -186.88 & 2.54 & -1.38 & 69.192 \\
\hline 7788 & -187.28 & 2.47 & -1.47 & 69.198 \\
\hline 7787 & -187.53 & 2.53 & -1.33 & 69.201 \\
\hline 1291 & -187.85 & 2.46 & -1.34 & 69.206 \\
\hline 1290 & -188.01 & 2.51 & -1.25 & 69.209 \\
\hline 7764 & -188.14 & 2.50 & -1.11 & 69.211 \\
\hline 7765 & -188.4 & 2.58 & -1.71 & 69.215 \\
\hline 7766 & -188.65 & 2.50 & -1.17 & 69.218 \\
\hline 7767 & -189.05 & 2.51 & -1.45 & 69.224 \\
\hline 7770 & -189.12 & 2.52 & -1.46 & 69.225 \\
\hline 7768 & -189.33 & 2.46 & -1.07 & 69.229 \\
\hline 1289 & -189.59 & 2.60 & -1.08 & 69.232 \\
\hline 7769 & -189.87 & 2.45 & -0.99 & 69.237 \\
\hline 7771 & -190.37 & 2.57 & -1.57 & 69.244 \\
\hline 7772 & -190.62 & 2.58 & -1.00 & 69.248 \\
\hline 1288 & -190.68 & 2.58 & -1.08 & 69.249 \\
\hline 7773 & -190.97 & 2.51 & -1.35 & 69.253 \\
\hline 7774 & -191.18 & 2.58 & -1.06 & 69.256 \\
\hline 1287 & -191.24 & 2.45 & -1.03 & 69.257 \\
\hline 7762 & -191.58 & 2.54 & -0.89 & 69.262 \\
\hline
\end{tabular}




\begin{tabular}{|c|c|c|c|c|}
\hline 7761 & -191.87 & 2.54 & -1.40 & 69.267 \\
\hline 1286 & -192.22 & 2.55 & -1.29 & 69.272 \\
\hline 7758 & -192.27 & 2.46 & -1.36 & 69.273 \\
\hline 7760 & -192.28 & 2.47 & -1.48 & 69.273 \\
\hline 7763 & -192.35 & 2.54 & -0.92 & 69.274 \\
\hline 7759 & -192.53 & 2.51 & -1.15 & 69.277 \\
\hline 1285 & -193 & 2.44 & -0.59 & 69.284 \\
\hline 7757 & -193.21 & 2.54 & -1.49 & 69.287 \\
\hline 7756 & -193.46 & 2.49 & -1.35 & 69.291 \\
\hline 7755 & -193.72 & 2.42 & -1.42 & 69.295 \\
\hline 1284 & -194.04 & 2.55 & -0.94 & 69.299 \\
\hline 7754 & -194.12 & 2.43 & -1.46 & 69.301 \\
\hline 7753 & -194.38 & 2.42 & -1.14 & 69.304 \\
\hline 4012 & -194.4 & 2.43 & -1.37 & 69.305 \\
\hline 7752 & -194.63 & 2.47 & -1.32 & 69.308 \\
\hline 1283 & -194.84 & 2.55 & -1.06 & 69.311 \\
\hline 7750 & -195.2 & 2.50 & -1.59 & 69.317 \\
\hline 7749 & -195.47 & 2.47 & -1.17 & 69.321 \\
\hline 7748 & -195.72 & 2.50 & -1.10 & 69.325 \\
\hline 1282 & -196 & 2.52 & -1.35 & 69.329 \\
\hline 7747 & -196.17 & 2.43 & -1.08 & 69.331 \\
\hline 7746 & -196.44 & 2.47 & -1.22 & 69.335 \\
\hline 7745 & -196.68 & 2.33 & -1.43 & 69.339 \\
\hline 1281 & -196.93 & 2.49 & -1.06 & 69.343 \\
\hline 7786 & -197.09 & 2.43 & -1.41 & 69.345 \\
\hline 7785 & -197.35 & 2.37 & -1.37 & 69.349 \\
\hline 7784 & -197.62 & 2.42 & -1.17 & 69.353 \\
\hline 1280 & -197.87 & 2.43 & -0.98 & 69.357 \\
\hline 7783 & -198.03 & 2.60 & -1.01 & 69.359 \\
\hline 7782 & -198.26 & 2.49 & -1.54 & 69.363 \\
\hline 7781 & -198.54 & 2.50 & -1.33 & 69.367 \\
\hline 1279 & -198.78 & 2.50 & -1.02 & 69.371 \\
\hline 7744 & -198.99 & 2.45 & -0.80 & 69.374 \\
\hline 7743 & -199.21 & 2.43 & -0.83 & 69.377 \\
\hline 7742 & -199.48 & 2.43 & -0.93 & 69.381 \\
\hline 1278 & -199.73 & 2.55 & -1.02 & 69.385 \\
\hline 7741 & -200 & 2.45 & -0.69 & 69.389 \\
\hline 7740 & -200.25 & 2.55 & -0.73 & 69.393 \\
\hline 7739 & -200.5 & 2.44 & -0.72 & 69.396 \\
\hline 1277 & -200.75 & 2.52 & -1.07 & 69.400 \\
\hline 7738 & -200.87 & 2.48 & -1.10 & 69.402 \\
\hline 7737 & -201.13 & 2.44 & -0.95 & 69.406 \\
\hline 7736 & -201.39 & 2.51 & -0.77 & 69.410 \\
\hline 1276 & -201.66 & 2.57 & -0.75 & 69.414 \\
\hline 7735 & -201.8 & 2.56 & -0.66 & 69.416 \\
\hline 7734 & -202.04 & 2.47 & -1.02 & 69.421 \\
\hline 7733 & -202.29 & 2.43 & -0.95 & 69.426 \\
\hline 1275 & -202.59 & 2.36 & -1.00 & 69.432 \\
\hline
\end{tabular}




$\begin{array}{lrlll}7732 & -202.78 & 2.47 & -0.94 & 69.436 \\ 7730 & -203.3 & 2.38 & -1.07 & 69.446 \\ 7731 & -203.5 & 2.34 & -0.70 & 69.450 \\ 1274 & -203.55 & 2.30 & -0.77 & 69.451 \\ 7729 & -203.66 & 2.38 & -1.17 & 69.453 \\ 7728 & -203.89 & 2.48 & -1.14 & 69.458 \\ 4011 & -203.95 & 2.36 & -1.15 & 69.459 \\ 7727 & -204.14 & 2.33 & -1.23 & 69.463 \\ 1273 & -204.41 & 2.43 & -0.94 & 69.468 \\ 7726 & -204.65 & 2.42 & -1.13 & 69.473 \\ 7725 & -204.91 & 2.38 & -1.31 & 69.478 \\ 7724 & -205.15 & 2.35 & -1.41 & 69.483 \\ 1272 & -205.38 & 2.29 & -1.03 & 69.488 \\ 7723 & -205.64 & 2.20 & -1.28 & 69.493 \\ 7722 & -205.88 & 2.25 & -1.14 & 69.498 \\ 7721 & -206.13 & 2.35 & -1.14 & 69.503 \\ 7720 & -206.32 & 2.24 & -1.11 & 69.507 \\ 1271 & -206.38 & 2.49 & -0.97 & 69.508 \\ 7719 & -206.55 & 2.29 & -1.50 & 69.511 \\ 1270 & -206.81 & 2.26 & -0.98 & 69.516 \\ 7718 & -207.05 & 2.21 & -1.37 & 69.521 \\ 7717 & -207.31 & 2.16 & -1.38 & 69.526 \\ 7716 & -207.56 & 2.22 & -1.38 & 69.531 \\ 1269 & -207.81 & 2.37 & -0.93 & 69.536 \\ 7715 & -208.12 & 2.21 & -1.38 & 69.543 \\ 7714 & -208.37 & 2.20 & -1.49 & 69.548 \\ 7713 & -208.61 & 2.24 & -1.65 & 69.552 \\ 1268 & -208.86 & 2.32 & -1.01 & 69.558 \\ 7712 & -209.04 & 2.20 & -1.07 & 69.561 \\ 7711 & -209.28 & 2.26 & -1.08 & 69.566 \\ 7710 & -209.54 & 2.32 & -1.37 & 69.571 \\ 1267 & -209.78 & 2.39 & -0.86 & 69.576 \\ 7709 & -210.01 & 2.24 & -0.93 & 69.581 \\ 7708 & -210.27 & 2.31 & -1.13 & 69.586 \\ 7707 & -210.52 & 2.17 & -1.53 & 69.591 \\ 1266 & -210.77 & 2.34 & -0.94 & 69.596 \\ 7706 & -211.03 & 2.23 & -1.20 & 69.601 \\ 7705 & -211.28 & 2.31 & -1.24 & 69.606 \\ 7704 & -211.54 & 2.25 & -1.11 & 69.611 \\ 1265 & -211.76 & 2.31 & -1.09 & 69.616 \\ 7703 & -211.97 & 2.34 & -1.01 & 69.620 \\ 7702 & -212.22 & 2.26 & -0.99 & 69.625 \\ 7701 & -212.47 & 2.17 & -0.95 & 69.630 \\ 7699 & -212.57 & 2.21 & -0.95 & 69.632 \\ 1264 & -212.71 & 2.20 & -0.90 & 69.635 \\ 7700 & -212.9 & 2.28 & -0.87 & 69.638 \\ 7698 & -213.49 & 2.29 & -1.22 & 69.650 \\ 1263 & -213.69 & 2.31 & -0.97 & 69.654\end{array}$




$\begin{array}{lrlll}7697 & -213.81 & 2.28 & -1.06 & 69.657 \\ 7696 & -214.03 & 2.21 & -1.12 & 69.661 \\ 4010 & -214.1 & 2.42 & -1.23 & 69.663 \\ 7695 & -214.28 & 2.18 & -0.97 & 69.666 \\ 1262 & -214.6 & 2.34 & -0.76 & 69.673 \\ 7694 & -214.86 & 2.26 & -0.90 & 69.678 \\ 7693 & -215.12 & 2.25 & -1.14 & 69.683 \\ 7692 & -215.38 & 2.28 & -1.22 & 69.688 \\ 1261 & -215.62 & 2.35 & -0.89 & 69.693 \\ 7691 & -215.68 & 2.29 & -0.89 & 69.694 \\ 7690 & -215.93 & 2.16 & -0.93 & 69.699 \\ 7689 & -216.17 & 2.24 & -0.89 & 69.704 \\ 1260 & -216.5 & 2.25 & -0.87 & 69.711 \\ 7688 & -216.76 & 2.10 & -0.99 & 69.716 \\ 7687 & -217 & 2.19 & -1.03 & 69.721 \\ 7686 & -217.25 & 2.17 & -1.02 & 69.726 \\ 1259 & -217.5 & 2.38 & -0.86 & 69.731 \\ 7685 & -217.52 & 2.11 & -1.06 & 69.731 \\ 7684 & -217.79 & 2.14 & -0.75 & 69.736 \\ 7683 & -218.04 & 2.10 & -0.93 & 69.742 \\ 1258 & -218.3 & 2.16 & -0.82 & 69.747 \\ 7682 & -218.5 & 2.19 & -0.97 & 69.751 \\ 7681 & -218.74 & 2.14 & -0.88 & 69.756 \\ 7680 & -218.99 & 2.17 & -0.75 & 69.761 \\ 1257 & -219.29 & 2.27 & -0.98 & 69.767 \\ 7679 & -219.44 & 2.20 & -0.90 & 69.770 \\ 7678 & -219.7 & 2.19 & -0.89 & 69.775 \\ 1256 & -220.1 & 2.20 & -0.87 & 69.783 \\ 7677 & -220.11 & 2.16 & -0.89 & 69.783 \\ 7676 & -220.37 & 2.12 & -1.05 & 69.788 \\ 7675 & -220.62 & 2.20 & -0.85 & 69.793 \\ 1255 & -220.87 & 2.30 & -0.85 & 69.798 \\ 7674 & -221.11 & 2.08 & -1.10 & 69.803 \\ 7673 & -221.36 & 2.06 & -0.83 & 69.808 \\ 7672 & -221.61 & 2.13 & -0.92 & 69.813 \\ 1254 & -221.87 & 2.23 & -0.69 & 69.818 \\ 7671 & -222.07 & 2.23 & -0.81 & 69.822 \\ 7670 & -222.33 & 2.13 & -1.05 & 69.827 \\ 7669 & -222.57 & 2.16 & -1.03 & 69.832 \\ 1253 & -222.82 & 2.21 & -0.64 & 69.837 \\ 7668 & -223 & 2.14 & -0.91 & 69.841 \\ 7667 & -223.28 & 2.20 & -0.76 & 69.847 \\ 7666 & -223.51 & 2.19 & -0.73 & 69.851 \\ 1252 & -223.76 & 2.37 & -0.84 & 69.856 \\ 7665 & -223.92 & 2.27 & -0.75 & 69.859 \\ 7664 & -224.17 & 2.23 & -0.73 & 69.864 \\ 7663 & -224.42 & 2.23 & -0.64 & 69.869 \\ 1251 & -224.68 & 2.28 & -0.77 & 69.875\end{array}$




$\begin{array}{lllll}4008 & -224.7 & 2.09 & -0.93 & 69.875 \\ 7662 & -224.83 & 2.24 & -0.32 & 69.878 \\ 7661 & -225.08 & 2.24 & -0.94 & 69.883 \\ 7660 & -225.27 & 2.22 & -0.52 & 69.886 \\ 1250 & -225.58 & 2.34 & -1.06 & 69.893 \\ 7659 & -225.75 & 2.21 & -1.03 & 69.896 \\ 7658 & -226.01 & 2.25 & -0.53 & 69.901 \\ 7657 & -226.26 & 2.27 & -0.84 & 69.906 \\ 1249 & -226.53 & 2.13 & -1.03 & 69.912 \\ 7656 & -226.77 & 2.26 & -0.59 & 69.916 \\ 7655 & -227.02 & 2.19 & -0.80 & 69.921 \\ 7654 & -227.28 & 2.08 & -1.12 & 69.927 \\ 1248 & -227.52 & 2.05 & -1.24 & 69.932 \\ 7653 & -227.62 & 2.22 & -1.12 & 69.934 \\ 7652 & -227.87 & 2.20 & -1.16 & 69.939 \\ 7651 & -228.12 & 2.23 & -1.17 & 69.944 \\ 1247 & -228.46 & 2.27 & -0.90 & 69.950 \\ 7650 & -228.59 & 2.30 & -1.08 & 69.953 \\ 7649 & -228.84 & 2.25 & -0.90 & 69.958 \\ 7648 & -229.09 & 2.24 & -0.86 & 69.963 \\ 1246 & -229.33 & 2.43 & -1.09 & 69.968 \\ 7647 & -229.52 & 2.23 & -0.92 & 69.972 \\ 7646 & -229.77 & 2.26 & -0.76 & 69.977 \\ 7645 & -230.02 & 2.21 & -1.42 & 69.982 \\ 1245 & -230.27 & 2.15 & -1.26 & 69.987 \\ 7644 & -230.46 & 2.28 & -0.98 & 69.990 \\ 7643 & -230.71 & 2.24 & -1.06 & 69.995 \\ 7642 & -230.96 & 2.31 & -1.04 & 70.000 \\ 1244 & -231.26 & 2.29 & -0.86 & 70.006 \\ 7641 & -231.44 & 2.33 & -0.29 & 70.010 \\ 7640 & -231.7 & 2.29 & -1.39 & 70.015 \\ 7639 & -231.95 & 2.22 & -1.11 & 70.020 \\ 1243 & -232.18 & 2.07 & -1.10 & 70.025 \\ 7638 & -232.44 & 2.32 & -0.82 & 70.030 \\ 7637 & -232.68 & 2.27 & -0.91 & 70.035 \\ 7636 & -232.92 & 2.29 & -0.97 & 70.040 \\ 1242 & -233.15 & 2.26 & -1.18 & 70.044 \\ 7635 & -233.21 & 2.36 & -1.06 & 70.046 \\ 7634 & -233.47 & 2.33 & -0.81 & 70.051 \\ 7633 & -233.72 & 2.33 & -1.04 & 70.056 \\ 1241 & -233.95 & 2.29 & -0.94 & 70.060 \\ 7632 & -234.15 & 2.27 & -1.29 & 70.064 \\ 7631 & -234.4 & 2.20 & -1.07 & 70.069 \\ 7630 & -234.66 & 2.29 & -1.20 & 70.075 \\ 1240 & -234.89 & 2.14 & -1.00 & 70.079 \\ 7629 & -235.17 & 2.12 & -0.97 & 70.085 \\ 7628 & -235.41 & 2.21 & -0.92 & 70.090 \\ 7627 & -235.66 & 2.21 & -0.74 & 70.095\end{array}$




$\begin{array}{lrlll}1239 & -235.91 & 2.17 & -0.95 & 70.100 \\ 7626 & -236.06 & 2.21 & -0.93 & 70.103 \\ 7625 & -236.31 & 2.26 & -0.81 & 70.108 \\ 7624 & -236.56 & 2.17 & -0.88 & 70.113 \\ 1238 & -236.8 & 2.18 & -1.15 & 70.118 \\ 7623 & -237 & 2.16 & -0.82 & 70.122 \\ 7622 & -237.25 & 2.24 & -0.90 & 70.127 \\ 7621 & -237.51 & 2.22 & -0.88 & 70.132 \\ 1237 & -237.74 & 2.08 & -1.39 & 70.136 \\ 7620 & -237.88 & 2.24 & -0.88 & 70.139 \\ 7619 & -238.13 & 2.22 & -1.01 & 70.144 \\ 7618 & -238.37 & 2.21 & -1.01 & 70.149 \\ 1236 & -238.71 & 2.18 & -1.01 & 70.156 \\ 7617 & -238.91 & 2.22 & -0.93 & 70.160 \\ 7616 & -239.16 & 2.26 & -0.77 & 70.165 \\ 7615 & -239.41 & 2.29 & -0.90 & 70.170 \\ 1235 & -239.66 & 2.23 & -0.93 & 70.175 \\ 7614 & -239.87 & 2.25 & -0.88 & 70.179 \\ 7613 & -240.12 & 2.31 & -0.80 & 70.184 \\ 1234 & -240.43 & 2.10 & -1.20 & 70.190 \\ 7612 & -240.63 & 2.31 & -0.87 & 70.194 \\ 7611 & -240.88 & 2.31 & -0.93 & 70.199 \\ 4009 & -241.1 & 2.20 & -1.14 & 70.204 \\ 7610 & -241.13 & 2.22 & -1.04 & 70.204 \\ 1233 & -241.38 & 2.12 & -0.99 & 70.209 \\ 7609 & -241.55 & 2.36 & -1.15 & 70.213 \\ 7608 & -241.8 & 2.29 & -0.68 & 70.218 \\ 7607 & -242.05 & 2.22 & -0.83 & 70.223 \\ 1232 & -242.3 & 2.26 & -1.46 & 70.228 \\ 7606 & -242.43 & 2.27 & -0.89 & 70.230 \\ 7605 & -242.67 & 2.23 & -0.94 & 70.235 \\ 7604 & -242.92 & 2.17 & -0.98 & 70.240 \\ 1231 & -243.09 & 2.24 & -1.20 & 70.244 \\ 7603 & -243.32 & 2.28 & -0.84 & 70.248 \\ 7602 & -243.57 & 2.26 & -0.93 & 70.253 \\ 7601 & -243.82 & 2.20 & -0.93 & 70.258 \\ 4005 & -244.05 & 2.23 & -0.66 & 70.263 \\ 1230 & -244.14 & 2.05 & -1.28 & 70.265 \\ 7600 & -244.21 & 2.30 & -0.77 & 70.266 \\ 7599 & -244.47 & 2.10 & -1.06 & 70.271 \\ 7598 & -244.72 & 2.19 & -0.95 & 70.276 \\ 1229 & -244.95 & 2.17 & -1.18 & 70.281 \\ 7597 & -245.09 & 2.22 & -1.03 & 70.284 \\ 7596 & -245.34 & 2.22 & -1.14 & 70.289 \\ 7595 & -245.59 & 2.20 & -1.00 & 70.294 \\ 1228 & -245.84 & 2.23 & -0.35 & 70.299 \\ 7594 & -246.13 & 2.23 & -0.98 & 70.305 \\ 7593 & -246.38 & 2.18 & -0.91 & 70.310\end{array}$




$\begin{array}{lrlll}7592 & -246.63 & 2.16 & -1.14 & 70.315 \\ 1227 & -246.86 & 2.11 & -1.12 & 70.319 \\ 7591 & -247.09 & 2.24 & -0.96 & 70.324 \\ 7590 & -247.33 & 2.14 & -1.04 & 70.329 \\ 7589 & -247.57 & 2.26 & -1.09 & 70.333 \\ 1226 & -247.84 & 2.29 & -0.76 & 70.339 \\ 7588 & -247.98 & 2.27 & -0.93 & 70.342 \\ 7587 & -248.23 & 2.27 & -0.86 & 70.347 \\ 7586 & -248.48 & 2.18 & -0.91 & 70.352 \\ 1225 & -248.73 & 2.24 & -0.82 & 70.357 \\ 7585 & -248.82 & 2.23 & -0.97 & 70.358 \\ 7584 & -249.07 & 2.12 & -0.39 & 70.363 \\ 7583 & -249.33 & 2.13 & -1.23 & 70.369 \\ 1224 & -249.63 & 2.12 & -0.87 & 70.375 \\ 7582 & -249.66 & 2.20 & -0.93 & 70.375 \\ 7581 & -249.91 & 2.12 & -1.16 & 70.380 \\ 7580 & -250.15 & 2.11 & -0.92 & 70.385 \\ 7579 & -250.4 & 2.07 & -0.93 & 70.390 \\ 1223 & -250.53 & 2.14 & -0.83 & 70.393 \\ 7578 & -250.67 & 2.14 & -0.99 & 70.396 \\ 7577 & -250.93 & 2.14 & -0.83 & 70.401 \\ 7576 & -251.18 & 2.02 & -1.11 & 70.406 \\ 1222 & -251.43 & 2.22 & -0.57 & 70.411 \\ 7575 & -251.55 & 2.03 & -0.95 & 70.413 \\ 7574 & -251.8 & 2.10 & -1.29 & 70.418 \\ 7573 & -252.05 & 2.05 & -0.97 & 70.423 \\ 1221 & -252.31 & 2.18 & -0.66 & 70.428 \\ 7572 & -252.53 & 2.14 & -1.07 & 70.433 \\ 7571 & -252.78 & 2.10 & -1.69 & 70.438 \\ 7570 & -253.04 & 2.11 & -1.39 & 70.443 \\ 1220 & -253.27 & 2.06 & -1.03 & 70.448 \\ 7569 & -253.51 & 2.12 & -0.93 & 70.452 \\ 7568 & -253.77 & 2.11 & -0.09 & 70.458 \\ 7567 & -254.03 & 2.15 & -0.66 & 70.463 \\ 4006 & -254.1 & 2.19 & -1.05 & 70.464 \\ 1219 & -254.27 & 2.19 & -0.67 & 70.468 \\ 7566 & -254.36 & 2.25 & -1.32 & 70.469 \\ 7565 & -254.62 & 2.24 & -0.77 & 70.475 \\ 7564 & -254.9 & 2.18 & -1.09 & 70.480 \\ 1218 & -255.2 & 2.09 & -0.88 & 70.486 \\ 7563 & -255.38 & 2.16 & -1.13 & 70.490 \\ 7562 & -255.64 & 2.21 & -0.80 & 70.495 \\ 7561 & -255.87 & 2.25 & -0.74 & 70.500 \\ 1217 & -256.13 & 2.14 & -0.86 & 70.505 \\ 7560 & -256.29 & 2.21 & -0.71 & 70.508 \\ 7559 & -256.56 & 2.14 & -0.89 & 70.514 \\ 7558 & -256.81 & 2.11 & -0.81 & 70.519 \\ 1216 & -257.12 & 2.03 & -1.14 & 70.525\end{array}$




$\begin{array}{lrlll}7557 & -257.19 & 2.07 & -0.84 & 70.526 \\ 7556 & -257.46 & 2.22 & -0.63 & 70.532 \\ 7555 & -257.72 & 2.13 & -0.65 & 70.537 \\ 1215 & -258.05 & 2.12 & -0.96 & 70.543 \\ 7554 & -258.15 & 2.23 & -0.55 & 70.545 \\ 7553 & -258.41 & 2.18 & -0.78 & 70.551 \\ 7552 & -258.64 & 2.11 & -0.89 & 70.555 \\ 1214 & -258.91 & 2.09 & -0.75 & 70.561 \\ 7551 & -259.1 & 2.09 & -1.72 & 70.564 \\ 7550 & -259.34 & 2.07 & -0.89 & 70.569 \\ 1213 & -259.84 & 2.03 & -1.01 & 70.579 \\ 7548 & -260.08 & 2.11 & -0.82 & 70.584 \\ 7547 & -260.33 & 2.09 & -0.89 & 70.589 \\ 7546 & -260.58 & 2.04 & -0.95 & 70.594 \\ 1212 & -260.82 & 2.01 & -0.78 & 70.599 \\ 7545 & -261.03 & 2.12 & -1.45 & 70.603 \\ 7544 & -261.26 & 1.99 & -0.82 & 70.608 \\ 7543 & -261.51 & 2.04 & -0.15 & 70.613 \\ 1211 & -261.77 & 2.16 & -0.83 & 70.618 \\ 7542 & -261.9 & 2.16 & -1.24 & 70.621 \\ 7541 & -262.17 & 2.06 & -0.72 & 70.626 \\ 7540 & -262.43 & 2.14 & -0.80 & 70.631 \\ 1210 & -262.74 & 2.03 & -0.80 & 70.637 \\ 7539 & -262.89 & 2.11 & -0.93 & 70.640 \\ 7538 & -263.13 & 2.17 & -0.71 & 70.645 \\ 7537 & -263.38 & 2.10 & -0.81 & 70.650 \\ 1209 & -263.63 & 2.01 & -0.77 & 70.655 \\ 7536 & -263.81 & 2.16 & -0.62 & 70.659 \\ 4004 & -263.93 & 2.03 & -0.76 & 70.661 \\ 7535 & -264.07 & 2.08 & -0.73 & 70.664 \\ 7534 & -264.32 & 2.10 & -0.65 & 70.669 \\ 1208 & -264.58 & 2.04 & -0.74 & 70.674 \\ 7533 & -264.77 & 2.04 & -0.76 & 70.678 \\ 7532 & -265.02 & 2.03 & -0.87 & 70.683 \\ 7532 & -265.02 & 2.06 & -1.52 & 70.683 \\ 7531 & -265.26 & 2.07 & -0.84 & 70.688 \\ 1207 & -265.49 & 2.11 & -0.92 & 70.693 \\ 7530 & -265.63 & 2.13 & -0.93 & 70.695 \\ 1206 & -265.87 & 2.03 & -1.00 & 70.700 \\ 7529 & -266.08 & 2.09 & -0.54 & 70.704 \\ 7528 & -266.49 & 1.96 & -1.40 & 70.713 \\ 7527 & -266.5 & 2.05 & -0.59 & 70.713 \\ 7526 & -266.78 & 2.00 & -0.76 & 70.718 \\ 7525 & -267.11 & 1.98 & -0.96 & 70.725 \\ 1205 & -267.31 & 2.01 & -0.98 & 70.729 \\ 7524 & -267.49 & 2.10 & -0.75 & 70.733 \\ 7523 & -267.75 & 1.96 & -0.75 & 70.738 \\ 1204 & -268.01 & 1.87 & -0.93 & 70.743\end{array}$




$\begin{array}{lrlll}7522 & -268.32 & 1.93 & -0.82 & 70.749 \\ 7521 & -268.57 & 1.98 & -0.67 & 70.754 \\ 7520 & -268.85 & 1.94 & -0.69 & 70.760 \\ 1203 & -269.11 & 1.83 & -1.01 & 70.765 \\ 7519 & -269.13 & 2.06 & -0.97 & 70.766 \\ 7518 & -269.36 & 2.06 & -0.78 & 70.770 \\ 7517 & -269.6 & 1.97 & -0.98 & 70.775 \\ 1202 & -269.85 & 1.75 & -1.08 & 70.780 \\ 7516 & -270.09 & 1.92 & -0.81 & 70.785 \\ 7515 & -270.34 & 1.99 & -1.06 & 70.790 \\ 7514 & -270.59 & 1.99 & -0.81 & 70.795 \\ 1201 & -270.85 & 1.84 & -1.09 & 70.800 \\ 7513 & -271.06 & 1.93 & -0.73 & 70.804 \\ 7512 & -271.3 & 2.06 & -0.77 & 70.809 \\ 7511 & -271.55 & 1.97 & -0.90 & 70.814 \\ 1200 & -271.8 & 1.85 & -0.88 & 70.819 \\ 7510 & -272.01 & 2.01 & -0.66 & 70.823 \\ 7509 & -272.28 & 1.93 & -1.63 & 70.829 \\ 7508 & -272.53 & 1.88 & -0.96 & 70.834 \\ 7507 & -272.72 & 1.99 & -1.30 & 70.837 \\ 1199 & -272.79 & 1.74 & -1.35 & 70.839 \\ 7506 & -273.13 & 2.02 & -1.30 & 70.846 \\ 7505 & -273.38 & 1.98 & -1.01 & 70.851 \\ 1198 & -273.71 & 2.03 & -0.94 & 70.857 \\ 7504 & -273.82 & 1.93 & -1.09 & 70.860 \\ 7503 & -274.08 & 1.90 & -1.13 & 70.865 \\ 7502 & -274.33 & 1.90 & -1.16 & 70.870 \\ 4003 & -274.55 & 1.96 & -0.74 & 70.874 \\ 1197 & -274.62 & 2.05 & -0.52 & 70.876 \\ 7501 & -274.82 & 1.96 & -0.87 & 70.880 \\ 7500 & -275.07 & 1.85 & -0.80 & 70.885 \\ 7499 & -275.32 & 1.87 & -0.73 & 70.890 \\ 1196 & -275.54 & 1.91 & -1.09 & 70.894 \\ 7498 & -275.68 & 1.94 & -0.65 & 70.897 \\ 7497 & -275.94 & 1.98 & -0.71 & 70.902 \\ 7496 & -276.18 & 1.92 & -0.66 & 70.907 \\ 1195 & -276.46 & 1.83 & -1.09 & 70.912 \\ 7495 & -276.5 & 2.04 & -0.75 & 70.913 \\ 7494 & -276.76 & 2.04 & -0.78 & 70.918 \\ 7493 & -277.01 & 1.92 & -0.86 & 70.923 \\ 1194 & -277.34 & 1.87 & -0.79 & 70.930 \\ 7492 & -277.51 & 2.00 & -0.92 & 70.933 \\ 7491 & -277.77 & 1.92 & -0.72 & 70.939 \\ 7490 & -278.03 & 1.97 & -0.83 & 70.944 \\ 1193 & -278.33 & 2.10 & -0.61 & 70.950 \\ 7489 & -278.4 & 2.01 & -0.41 & 70.951 \\ 7488 & -278.64 & 1.95 & -0.72 & 70.956 \\ 7487 & -278.9 & 2.06 & -0.43 & 70.961\end{array}$




$\begin{array}{lrlll}1192 & -279.19 & 1.97 & -0.91 & 70.967 \\ 7486 & -279.39 & 2.01 & -0.76 & 70.971 \\ 7485 & -279.66 & 1.94 & -0.91 & 70.977 \\ 1191 & -279.97 & 1.83 & -0.98 & 70.983 \\ 7484 & -280.3 & 2.04 & -0.89 & 70.989 \\ 7483 & -280.55 & 2.02 & -0.76 & 70.994 \\ 7482 & -280.81 & 1.95 & -1.02 & 71.000 \\ 1190 & -281.04 & 2.10 & -0.50 & 71.004 \\ 7481 & -281.22 & 1.97 & -0.99 & 71.008 \\ 7480 & -281.46 & 2.01 & -0.90 & 71.013 \\ 7479 & -281.72 & 2.03 & -0.82 & 71.018 \\ 1189 & -281.97 & 1.83 & -0.95 & 71.023 \\ 7478 & -282.22 & 1.96 & -0.82 & 71.028 \\ 7477 & -282.47 & 1.97 & -0.80 & 71.033 \\ 7476 & -282.72 & 1.99 & -0.93 & 71.038 \\ 1188 & -282.96 & 2.00 & -0.83 & 71.043 \\ 7475 & -283.19 & 1.97 & -1.02 & 71.047 \\ 7474 & -283.45 & 1.84 & -1.15 & 71.053 \\ 7473 & -283.69 & 1.84 & -0.85 & 71.057 \\ 1187 & -283.915 & 1.83 & -0.81 & 71.062 \\ 7472 & -284 & 1.90 & -0.78 & 71.064 \\ 4002 & -284.05 & 1.89 & -0.97 & 71.065 \\ 7471 & -284.31 & 2.01 & -0.97 & 71.070 \\ 7470 & -284.56 & 1.83 & -0.76 & 71.075 \\ 1186 & -284.81 & 1.89 & -1.16 & 71.080 \\ 7469 & -285.05 & 1.98 & -0.84 & 71.085 \\ 7468 & -285.32 & 1.96 & -0.80 & 71.090 \\ 7467 & -285.56 & 1.89 & -0.85 & 71.095 \\ 1185 & -285.825 & 1.82 & -1.03 & 71.100 \\ 7466 & -285.98 & 1.94 & -0.85 & 71.103 \\ 7465 & -286.24 & 1.78 & -0.90 & 71.108 \\ 7464 & -286.49 & 1.79 & -0.85 & 71.113 \\ 1184 & -286.8 & 1.85 & -0.90 & 71.120 \\ 7463 & -286.93 & 1.86 & -0.76 & 71.122 \\ 7462 & -287.2 & 1.91 & -1.03 & 71.128 \\ 7461 & -287.47 & 1.99 & -1.02 & 71.133 \\ 1183 & -287.77 & 1.91 & -0.91 & 71.139 \\ 7460 & -287.89 & 1.91 & -0.99 & 71.142 \\ 7459 & -288.13 & 1.91 & -0.98 & 71.146 \\ 7458 & -288.37 & 1.91 & -0.21 & 71.151 \\ 1182 & -288.64 & 1.97 & -0.92 & 71.157 \\ 7457 & -289.05 & 2.00 & -0.91 & 71.165 \\ 7456 & -289.3 & 1.98 & -0.92 & 71.170 \\ 1181 & -289.67 & 1.86 & -1.08 & 71.177 \\ 7455 & -289.82 & 1.99 & -0.85 & 71.180 \\ 7454 & -290.08 & 1.93 & -1.00 & 71.185 \\ 7453 & -290.33 & 1.93 & -1.09 & 71.190 \\ 1180 & -290.66 & 1.93 & -1.08 & 71.197\end{array}$




$\begin{array}{lllll}7452 & -290.74 & 1.95 & -0.88 & 71.199 \\ 7451 & -290.95 & 1.92 & -1.15 & 71.203 \\ 7450 & -291.13 & 1.94 & -0.95 & 71.206 \\ 1179 & -291.44 & 1.91 & -1.21 & 71.213 \\ 7449 & -291.46 & 1.98 & -1.20 & 71.213 \\ 7448 & -291.73 & 1.99 & -1.29 & 71.218 \\ 7447 & -291.97 & 1.94 & -1.14 & 71.223 \\ 1178 & -292.31 & 2.07 & -1.19 & 71.230 \\ 7446 & -292.57 & 1.84 & -1.15 & 71.235 \\ 7445 & -292.82 & 1.96 & -1.27 & 71.240 \\ 1177 & -293.26 & 1.93 & -0.93 & 71.249 \\ 7444 & -293.46 & 1.98 & -1.29 & 71.253 \\ 1176 & -294.13 & 2.13 & -0.95 & 71.267 \\ 7443 & -294.28 & 2.00 & -0.88 & 71.270 \\ 7442 & -294.52 & 1.96 & -1.03 & 71.274 \\ 7441 & -294.74 & 1.96 & -0.85 & 71.279 \\ 4001 & -294.91 & 2.03 & -1.04 & 71.282 \\ 1175 & -295.04 & 1.99 & -0.93 & 71.285 \\ 7440 & -295.15 & 2.03 & -1.06 & 71.287 \\ 7439 & -295.42 & 2.08 & -1.01 & 71.292 \\ 7438 & -295.66 & 2.06 & -1.22 & 71.297 \\ 1174 & -295.93 & 2.19 & -1.08 & 71.303 \\ 7437 & -296.14 & 1.99 & -1.32 & 71.307 \\ 7436 & -296.37 & 2.01 & -0.98 & 71.311 \\ 7435 & -296.57 & 2.04 & -1.00 & 71.315 \\ 1173 & -296.64 & 1.79 & -1.59 & 71.317 \\ 7434 & -296.98 & 1.92 & -0.96 & 71.324 \\ 7433 & -297.23 & 2.10 & -1.06 & 71.329 \\ 7432 & -297.49 & 2.11 & -0.93 & 71.334 \\ 1172 & -297.69 & 2.05 & -0.95 & 71.338 \\ 7431 & -297.97 & 2.05 & -1.19 & 71.344 \\ 7430 & -298.49 & 2.06 & -1.19 & 71.354 \\ 1171 & -298.66 & 1.78 & -1.41 & 71.357 \\ 7429 & -298.89 & 2.14 & -0.93 & 71.362 \\ 7428 & -299.16 & 2.13 & -0.97 & 71.367 \\ 7427 & -299.41 & 2.11 & -1.01 & 71.372 \\ 1170 & -299.63 & 2.00 & -0.96 & 71.377 \\ 7426 & -299.89 & 2.02 & -0.85 & 71.382 \\ 7425 & -300.15 & 2.00 & -0.99 & 71.387 \\ 1169 & -300.27 & 2.00 & -1.19 & 71.390 \\ 7424 & -300.69 & 2.00 & -1.18 & 71.398 \\ 7423 & -300.94 & 2.00 & -1.07 & 71.403 \\ 7422 & -301.2 & 1.97 & -0.96 & 71.408 \\ 1168 & -301.45 & 1.89 & -1.16 & 71.413 \\ 7421 & -301.66 & 1.92 & -0.92 & 71.418 \\ 7420 & -301.89 & 1.98 & -0.99 & 71.422 \\ 7419 & -302.14 & 1.97 & -0.89 & 71.427 \\ 1167 & -302.41 & 1.95 & -1.28 & 71.433\end{array}$




$\begin{array}{lllll}7418 & -302.79 & 2.12 & -1.38 & 71.440 \\ 7417 & -302.96 & 2.05 & -0.99 & 71.444 \\ 1166 & -303.39 & 1.96 & -1.16 & 71.452 \\ 7416 & -303.47 & 1.99 & -0.10 & 71.454 \\ 7415 & -303.73 & 2.02 & -0.59 & 71.459 \\ 7414 & -303.98 & 2.11 & -1.66 & 71.464 \\ 1165 & -304.31 & 1.95 & -1.22 & 71.471 \\ 7413 & -304.48 & 2.01 & -1.70 & 71.474 \\ 7412 & -304.69 & 1.99 & -1.66 & 71.478 \\ 7411 & -304.94 & 1.98 & -0.91 & 71.483 \\ 1164 & -305.29 & 1.91 & -1.17 & 71.490 \\ 7410 & -305.41 & 1.99 & -1.09 & 71.493 \\ 7409 & -305.89 & 1.98 & -1.13 & 71.502 \\ 1163 & -306.29 & 2.00 & -1.17 & 71.510 \\ 7408 & -306.34 & 1.95 & -0.89 & 71.511 \\ 7407 & -306.61 & 1.96 & -0.85 & 71.517 \\ 1162 & -307.05 & 1.84 & -1.07 & 71.526 \\ 7406 & -307.35 & 2.00 & -1.04 & 71.532 \\ 7405 & -307.82 & 2.09 & -1.00 & 71.541 \\ 1161 & -308.03 & 2.00 & -1.20 & 71.545 \\ 7404 & -308.22 & 2.06 & -1.27 & 71.549 \\ 7403 & -308.47 & 2.01 & -0.96 & 71.565 \\ 1160 & -308.84 & 1.95 & -1.14 & 71.588 \\ 7402 & -308.99 & 1.94 & -1.14 & 71.598 \\ 7401 & -309.21 & 1.90 & -1.35 & 71.612 \\ 7400 & -309.49 & 2.00 & -1.25 & 71.630 \\ 1159 & -309.72 & 1.82 & -1.50 & 71.644 \\ 7399 & -309.85 & 1.93 & -1.29 & 71.653 \\ 7398 & -310.1 & 1.88 & -1.18 & 71.669 \\ 7397 & -310.31 & 1.94 & -1.43 & 71.682 \\ 1158 & -310.76 & 1.85 & -1.32 & 71.711 \\ 7396 & -310.84 & 1.89 & -1.29 & 71.716 \\ 7395 & -311.29 & 1.77 & -1.40 & 71.744 \\ 1157 & -311.69 & 1.73 & -1.25 & 71.770 \\ 7394 & -311.89 & 1.80 & -1.40 & 71.783 \\ 7393 & -312.15 & 1.86 & -1.37 & 71.799 \\ 7392 & -312.23 & 1.92 & -1.26 & 71.804 \\ 1156 & -312.57 & 1.88 & -1.33 & 71.826 \\ 7391 & -312.66 & 2.02 & -1.61 & 71.832 \\ 7390 & -312.9 & 1.86 & -1.88 & 71.847 \\ 7389 & -313.14 & 1.81 & -1.22 & 71.862 \\ 7388 & -313.33 & 1.89 & -0.74 & 71.874 \\ 1155 & -313.61 & 1.82 & -1.43 & 71.892 \\ 7387 & -313.72 & 1.89 & -1.18 & 71.899 \\ 7386 & -313.94 & 1.81 & -1.82 & 71.913 \\ 7385 & -314.17 & 1.83 & -1.61 & 71.928 \\ 1154 & -314.45 & 1.84 & -1.39 & 71.945 \\ 7384 & -314.87 & 1.89 & -1.17 & 71.972\end{array}$




$\begin{array}{lllll}7383 & -315.07 & 1.79 & -1.13 & 71.985 \\ 1153 & -315.41 & 1.91 & -1.50 & 72.007 \\ 7382 & -315.49 & 1.83 & -1.52 & 72.012 \\ 7381 & -315.93 & 1.86 & -1.56 & 72.040 \\ 7380 & -316.15 & 1.84 & -1.45 & 72.054 \\ 1152 & -316.35 & 1.75 & -1.66 & 72.066 \\ 7379 & -317.01 & 1.80 & -1.39 & 72.108 \\ 1151 & -317.28 & 1.72 & -1.52 & 72.126 \\ 7378 & -317.68 & 1.78 & -1.31 & 72.151 \\ 7377 & -317.91 & 1.88 & -1.35 & 72.155 \\ 1150 & -318.24 & 1.82 & -1.55 & 72.161 \\ 7376 & -318.3 & 1.71 & -1.38 & 72.162 \\ 7375 & -318.77 & 1.70 & -1.30 & 72.170 \\ 1149 & -319.115 & 1.92 & -1.20 & 72.176 \\ 7374 & -319.25 & 1.96 & -1.22 & 72.179 \\ 7373 & -319.65 & 1.90 & -1.19 & 72.186 \\ 7372 & -319.85 & 1.90 & -1.10 & 72.189 \\ 1148 & -320.09 & 1.90 & -0.98 & 72.194 \\ 7371 & -320.28 & 1.90 & -1.35 & 72.197 \\ 7370 & -320.53 & 1.79 & -1.72 & 72.201 \\ 7369 & -320.99 & 2.10 & -1.65 & 72.210 \\ 1147 & -321.11 & 1.97 & -1.27 & 72.212 \\ 7368 & -321.24 & 1.91 & -1.34 & 72.214 \\ 7367 & -321.49 & 1.98 & -1.19 & 72.218 \\ 1146 & -321.76 & 1.94 & -1.09 & 72.223 \\ 7366 & -321.98 & 2.00 & -1.26 & 72.227 \\ 7365 & -322.49 & 1.86 & -1.55 & 72.236 \\ 1145 & -322.71 & 1.74 & -1.40 & 72.240 \\ 7364 & -323.16 & 1.90 & -1.28 & 72.248 \\ 7363 & -323.38 & 1.97 & -1.49 & 72.252 \\ 1144 & -323.68 & 2.05 & -1.46 & 72.257 \\ 1143 & -323.86 & 1.78 & -1.46 & 72.260 \\ 7362 & -324.09 & 1.99 & -1.42 & 72.264 \\ 7361 & -324.34 & 1.84 & -1.29 & 72.269 \\ 1142 & -324.77 & 1.85 & -1.39 & 72.277 \\ 7360 & -324.99 & 1.84 & -1.25 & 72.280 \\ 7359 & -325.45 & 1.91 & -1.36 & 72.289 \\ 1141 & -325.77 & 1.86 & -1.25 & 72.294 \\ 7358 & -325.96 & 1.83 & -1.39 & 72.298 \\ 7357 & -326.42 & 2.04 & -1.36 & 72.306 \\ 1140 & -326.72 & 1.98 & -1.53 & 72.311 \\ 7356 & -326.88 & 1.89 & -1.38 & 72.314 \\ 1139 & -327.66 & 1.83 & -1.76 & 72.328 \\ 7355 & -327.72 & 1.92 & -1.91 & 72.329 \\ 7354 & -328.1 & 1.95 & -1.61 & 72.335 \\ 1138 & -328.59 & 2.02 & -1.62 & 72.344 \\ 7353 & -328.71 & 1.92 & -1.42 & 72.346 \\ 1137 & -329.47 & 1.95 & -1.61 & 72.360\end{array}$




$\begin{array}{lrlll}7351 & -329.7 & 1.80 & -1.49 & 72.364 \\ 7350 & -330.12 & 1.94 & -1.34 & 72.371 \\ 1136 & -330.29 & 1.99 & -1.76 & 72.374 \\ 7349 & -330.68 & 1.96 & -1.17 & 72.381 \\ 7348 & -331.04 & 2.05 & -1.25 & 72.388 \\ 1135 & -331.26 & 2.04 & -1.02 & 72.391 \\ 7347 & -331.43 & 2.16 & -1.10 & 72.394 \\ 7346 & -331.68 & 2.14 & -1.10 & 72.399 \\ 7345 & -331.97 & 2.20 & -1.28 & 72.404 \\ 1134 & -332.24 & 2.13 & -1.47 & 72.409 \\ 7344 & -332.47 & 2.02 & -1.24 & 72.413 \\ 7343 & -332.74 & 1.98 & -1.38 & 72.418 \\ 7342 & -333.22 & 2.07 & -1.22 & 72.426 \\ 1133 & -333.25 & 2.07 & -1.16 & 72.427 \\ 7341 & -333.43 & 2.14 & -0.47 & 72.430 \\ 4007 & -334 & 2.15 & -0.98 & 72.440 \\ 1132 & -334.02 & 2.07 & -1.32 & 72.440 \\ 7340 & -334.31 & 2.13 & -1.47 & 72.445 \\ 7339 & -334.55 & 2.16 & -1.33 & 72.450 \\ 7338 & -334.97 & 2.11 & -1.48 & 72.457 \\ 1131 & -335.06 & 2.11 & -1.23 & 72.459 \\ 7337 & -335.22 & 2.20 & -1.40 & 72.462 \\ 7336 & -335.47 & 2.07 & -1.98 & 72.466 \\ 1130 & -335.79 & 2.15 & -1.38 & 72.472 \\ 7335 & -335.95 & 2.15 & -1.56 & 72.474 \\ 7334 & -336.41 & 2.12 & -1.62 & 72.483 \\ 1129 & -336.77 & 2.11 & -1.15 & 72.489 \\ 7333 & -336.91 & 2.14 & -1.42 & 72.491 \\ 7332 & -337.13 & 2.13 & -1.26 & 72.495 \\ 7331 & -337.47 & 2.13 & -1.28 & 72.501 \\ 1128 & -337.7 & 2.09 & -1.27 & 72.505 \\ 7330 & -337.93 & 2.24 & -1.06 & 72.510 \\ 7329 & -338.36 & 2.15 & -1.49 & 72.517 \\ 1127 & -338.63 & 2.26 & -1.31 & 72.522 \\ 7328 & -338.73 & 2.23 & -1.15 & 72.524 \\ 7327 & -339.15 & 2.11 & -1.45 & 72.531 \\ 1126 & -339.36 & 2.19 & -1.42 & 72.535 \\ 7326 & -339.55 & 2.28 & -1.10 & 72.538 \\ 7325 & -339.9 & 2.11 & -1.32 & 72.544 \\ 7324 & -340.15 & 2.27 & -1.50 & 72.549 \\ 1125 & -340.38 & 2.20 & -1.24 & 72.553 \\ 7323 & -340.66 & 2.21 & -1.35 & 72.558 \\ 7322 & -340.85 & 2.18 & -1.55 & 72.561 \\ 1124 & -341.27 & 2.10 & -1.42 & 72.569 \\ 7321 & -341.93 & 1.91 & -1.36 & 72.580 \\ 1123 & -342.19 & 1.96 & -1.22 & 72.585 \\ 7320 & -342.34 & 2.03 & -1.33 & 72.588 \\ 7319 & -342.55 & 2.13 & -1.38 & 72.591\end{array}$




$\begin{array}{lrlll}7318 & -343.01 & 2.14 & -1.42 & 72.599 \\ 1122 & -343.17 & 2.13 & -1.40 & 72.602 \\ 7317 & -343.3 & 2.10 & -1.29 & 72.605 \\ 7316 & -343.68 & 1.99 & -1.45 & 72.611 \\ 7315 & -343.9 & 2.01 & -1.28 & 72.615 \\ 1121 & -344.12 & 2.04 & -1.49 & 72.619 \\ 7314 & -344.75 & 2.11 & -1.74 & 72.630 \\ 1120 & -344.99 & 2.17 & -1.54 & 72.635 \\ 7313 & -345.22 & 2.15 & -1.51 & 72.639 \\ 7312 & -345.65 & 2.16 & -1.27 & 72.646 \\ 1119 & -345.85 & 2.18 & -1.50 & 72.650 \\ 7311 & -346.16 & 2.19 & -1.62 & 72.655 \\ 7310 & -346.63 & 2.23 & -1.67 & 72.664 \\ 1118 & -346.86 & 2.13 & -1.60 & 72.668 \\ 7309 & -347.42 & 2.13 & -1.43 & 72.678 \\ 1117 & -347.68 & 2.13 & -1.80 & 72.682 \\ 7308 & -348.09 & 2.03 & -1.88 & 72.689 \\ 1116 & -348.7 & 2.05 & -1.78 & 72.700 \\ 5542 & -348.87 & 1.96 & -1.19 & 72.703 \\ 7307 & -349.1 & 1.90 & -1.85 & 72.707 \\ 7306 & -349.51 & 1.99 & -1.90 & 72.715 \\ 1115 & -349.77 & 2.17 & -1.93 & 72.719 \\ 7305 & -350 & 2.03 & -1.49 & 72.723 \\ 7304 & -350.23 & 2.04 & -1.48 & 72.727 \\ 1114 & -350.72 & 1.96 & -1.90 & 72.736 \\ 7303 & -350.78 & 2.11 & -1.84 & 72.737 \\ 7302 & -351.02 & 2.22 & -1.65 & 72.741 \\ 7301 & -351.23 & 2.22 & -1.52 & 72.745 \\ 1113 & -351.6 & 2.13 & -1.73 & 72.752 \\ 7300 & -351.79 & 2.25 & -1.67 & 72.755 \\ 7299 & -352.28 & 2.27 & -1.65 & 72.764 \\ 1112 & -352.52 & 2.21 & -1.54 & 72.768 \\ 7298 & -353.06 & 2.22 & -1.70 & 72.777 \\ 7297 & -353.25 & 2.32 & -1.87 & 72.781 \\ 1111 & -353.45 & 2.17 & -1.83 & 72.784 \\ 7296 & -353.69 & 2.31 & -2.11 & 72.789 \\ 7295 & -354.13 & 2.23 & -1.84 & 72.796 \\ 1110 & -354.3 & 2.30 & -1.79 & 72.799 \\ 7293 & -354.89 & 2.29 & -1.35 & 72.810 \\ 1109 & -355.34 & 2.23 & -1.91 & 72.818 \\ 7292 & -355.6 & 2.33 & -1.59 & 72.822 \\ 7294 & -355.65 & 2.37 & -1.57 & 72.823 \\ 7291 & -356.04 & 2.39 & -1.51 & 72.830 \\ 1108 & -356.32 & 2.30 & -1.66 & 72.835 \\ 7290 & -356.52 & 2.27 & -1.61 & 72.839 \\ 7289 & -356.95 & 2.20 & -1.53 & 72.846 \\ 1107 & -357.21 & 2.24 & -1.36 & 72.851 \\ 7288 & -357.38 & 2.25 & -1.79 & 72.854\end{array}$




\begin{tabular}{|c|c|c|c|c|}
\hline 7287 & -357.58 & 2.13 & -1.42 & 72.857 \\
\hline 7286 & -357.95 & 2.36 & -1.46 & 72.864 \\
\hline 1106 & -358.15 & 2.31 & -1.45 & 72.868 \\
\hline 7285 & -358.64 & 2.49 & -1.96 & 72.876 \\
\hline 7284 & -358.89 & 2.46 & -1.50 & 72.881 \\
\hline 1105 & -359.14 & 2.56 & -1.55 & 72.885 \\
\hline 7283 & -359.29 & 2.50 & -1.49 & 72.888 \\
\hline 7282 & -359.53 & 2.42 & -1.55 & 72.892 \\
\hline 1104 & -360.01 & 2.53 & -1.53 & 72.900 \\
\hline 7281 & -360.21 & 2.50 & -1.59 & 72.904 \\
\hline 7280 & -360.74 & 2.53 & -1.68 & 72.913 \\
\hline 1103 & -360.99 & 2.57 & -1.62 & 72.918 \\
\hline 7279 & -361.14 & 2.66 & -1.64 & 72.921 \\
\hline 7278 & -361.5 & 2.54 & -1.85 & 72.927 \\
\hline 1102 & -361.75 & 2.38 & -1.76 & 72.931 \\
\hline 7277 & -362.01 & 2.54 & -1.99 & 72.936 \\
\hline 7276 & -362.38 & 2.63 & -1.60 & 72.942 \\
\hline 7275 & -362.58 & 2.58 & -2.07 & 72.946 \\
\hline 1101 & -362.8 & 2.70 & -1.97 & 72.947 \\
\hline 7274 & -363.12 & 2.53 & -1.59 & 72.950 \\
\hline 7273 & -363.38 & 2.43 & -1.24 & 72.951 \\
\hline 1100 & -363.82 & 2.43 & -1.81 & 72.954 \\
\hline 7272 & -363.97 & 2.43 & -1.70 & 72.955 \\
\hline 7271 & -364.2 & 2.43 & -1.84 & 72.957 \\
\hline 7270 & -364.57 & 2.45 & -1.59 & 72.960 \\
\hline 1099 & -364.75 & 2.43 & -1.94 & 72.961 \\
\hline 7269 & -364.83 & 2.53 & -1.65 & 72.961 \\
\hline 7268 & -365.03 & 2.50 & -1.73 & 72.963 \\
\hline 7267 & -365.48 & 2.56 & -1.81 & 72.966 \\
\hline 1098 & -365.65 & 2.56 & -1.59 & 72.967 \\
\hline 7266 & -365.93 & 2.51 & -1.68 & 72.969 \\
\hline 7265 & -366.16 & 2.45 & -1.65 & 72.970 \\
\hline 1097 & -366.55 & 2.54 & -2.08 & 72.973 \\
\hline 7264 & -366.8 & 2.43 & -1.79 & 72.975 \\
\hline 7263 & -367.08 & 2.48 & -1.81 & 72.977 \\
\hline 1096 & -367.5 & 2.44 & -2.12 & 72.979 \\
\hline 7262 & -367.7 & 2.41 & -1.75 & 72.981 \\
\hline 7261 & -367.91 & 2.39 & -2.28 & 72.982 \\
\hline 1095 & -368.52 & 2.27 & -2.07 & 72.986 \\
\hline 7260 & -368.69 & 2.43 & -2.35 & 72.988 \\
\hline 5510 & -368.94 & 2.42 & -0.54 & 72.989 \\
\hline 7259 & -369.18 & 2.48 & -2.46 & 72.991 \\
\hline 1094 & -369.41 & 2.39 & -2.03 & 72.992 \\
\hline 7258 & -369.88 & 2.32 & -2.04 & 72.996 \\
\hline 7257 & -370.16 & 2.42 & -1.71 & 72.998 \\
\hline 1093 & -370.39 & 2.55 & -2.49 & 72.999 \\
\hline 7256 & -370.53 & 2.54 & -1.82 & 73.000 \\
\hline 7255 & -370.78 & 2.57 & -1.47 & 73.002 \\
\hline
\end{tabular}




$\begin{array}{lrlll}1092 & -371.3 & 2.49 & -1.91 & 73.005 \\ 7254 & -371.64 & 2.52 & -1.47 & 73.008 \\ 7253 & -371.9 & 2.59 & -1.88 & 73.009 \\ 7252 & -372.18 & 2.44 & -2.02 & 73.011 \\ 7251 & -372.44 & 2.50 & -2.56 & 73.013 \\ 7250 & -372.66 & 2.47 & -1.61 & 73.015 \\ 1090 & -373.06 & 2.54 & -2.10 & 73.017 \\ 7249 & -373.22 & 2.52 & -1.66 & 73.018 \\ 7248 & -373.47 & 2.50 & -1.66 & 73.020 \\ 7247 & -373.73 & 2.57 & -1.58 & 73.022 \\ 1089 & -374.05 & 2.54 & -1.84 & 73.024 \\ 7246 & -374.52 & 2.47 & -1.56 & 73.027 \\ 1088 & -374.98 & 2.61 & -1.94 & 73.030 \\ 5501 & -375.08 & 2.51 & -1.13 & 73.031 \\ 7245 & -375.39 & 2.46 & -1.58 & 73.033 \\ 7244 & -375.62 & 2.50 & -1.69 & 73.035 \\ 1087 & -375.86 & 2.59 & -1.64 & 73.036 \\ 7243 & -376.09 & 2.55 & -1.41 & 73.038 \\ 7242 & -376.35 & 2.55 & -1.17 & 73.040 \\ 7241 & -376.59 & 2.44 & -1.35 & 73.041 \\ 1086 & -376.85 & 2.57 & -1.49 & 73.043 \\ 7240 & -377.04 & 2.50 & -1.67 & 73.044 \\ 7239 & -377.29 & 2.58 & -1.83 & 73.046 \\ 7238 & -377.54 & 2.47 & -1.71 & 73.048 \\ 1085 & -377.78 & 2.55 & -2.04 & 73.049 \\ 7237 & -378.09 & 2.47 & -1.72 & 73.051 \\ 7236 & -378.52 & 2.36 & -1.65 & 73.054 \\ 1084 & -378.72 & 2.32 & -2.25 & 73.056 \\ 7235 & -378.82 & 2.48 & -1.68 & 73.056 \\ 7234 & -379.08 & 2.47 & -1.20 & 73.058 \\ 1083 & -379.61 & 2.55 & -1.42 & 73.062 \\ 7233 & -379.7 & 2.44 & -1.80 & 73.062 \\ 7232 & -379.9 & 2.40 & -1.32 & 73.064 \\ 7231 & -380.12 & 2.44 & -1.91 & 73.065 \\ 7230 & -380.35 & 2.46 & -1.88 & 73.067 \\ 1082 & -380.6 & 2.26 & -2.13 & 73.069 \\ 7229 & -380.85 & 2.52 & -1.59 & 73.070 \\ 7228 & -381.09 & 2.37 & -1.68 & 73.072 \\ 1081 & -381.45 & 2.48 & -1.57 & 73.074 \\ 7227 & -381.63 & 2.36 & -1.59 & 73.076 \\ 7226 & -381.83 & 2.43 & -1.77 & 73.077 \\ 7225 & -382.11 & 2.44 & -1.84 & 73.079 \\ 1080 & -382.3 & 2.51 & -1.74 & 73.080 \\ 7224 & -382.38 & 2.38 & -1.68 & 73.081 \\ 7223 & -382.94 & 2.43 & -1.77 & 73.084 \\ 7222 & -383.13 & 2.43 & -1.67 & 73.086 \\ 1079 & -383.34 & 2.53 & -2.08 & 73.087 \\ 7221 & -383.68 & 2.38 & -1.50 & 73.089\end{array}$




$\begin{array}{lllll}7220 & -383.98 & 2.35 & -1.50 & 73.091 \\ 1078 & -384.18 & 2.44 & -1.80 & 73.093 \\ 7219 & -384.39 & 2.41 & -1.68 & 73.094 \\ 7218 & -384.93 & 2.35 & -1.75 & 73.098 \\ 1077 & -385.18 & 2.46 & -1.97 & 73.100 \\ 7217 & -385.35 & 2.43 & -1.84 & 73.101 \\ 7216 & -385.81 & 2.35 & -1.63 & 73.104 \\ 1076 & -386.05 & 2.36 & -1.79 & 73.106 \\ 7215 & -386.32 & 2.39 & -1.69 & 73.107 \\ 7214 & -386.77 & 2.32 & -1.64 & 73.110 \\ 1075 & -386.93 & 2.44 & -1.70 & 73.112 \\ 7213 & -387.02 & 2.37 & -1.91 & 73.112 \\ 7212 & -387.43 & 2.42 & -1.56 & 73.115 \\ 7211 & -387.65 & 2.45 & -1.82 & 73.116 \\ 1074 & -387.88 & 2.37 & -1.77 & 73.118 \\ 7210 & -388.14 & 2.34 & -1.80 & 73.120 \\ 7209 & -388.39 & 2.42 & -1.57 & 73.121 \\ 7208 & -388.64 & 2.47 & -1.69 & 73.123 \\ 1073 & -388.89 & 2.29 & -2.11 & 73.125 \\ 7207 & -389.22 & 2.33 & -1.66 & 73.127 \\ 7206 & -389.48 & 2.38 & -1.62 & 73.129 \\ 1072 & -389.71 & 2.48 & -1.91 & 73.130 \\ 7205 & -389.97 & 2.39 & -1.69 & 73.132 \\ 7204 & -390.21 & 2.39 & -1.69 & 73.134 \\ 7203 & -390.45 & 2.42 & -1.78 & 73.135 \\ 1071 & -390.73 & 2.21 & -1.66 & 73.137 \\ 7202 & -390.9 & 2.32 & -1.76 & 73.139 \\ 7201 & -391.18 & 2.37 & -1.94 & 73.140 \\ 1070 & -391.65 & 2.38 & -2.77 & 73.144 \\ 7200 & -391.77 & 2.37 & -2.17 & 73.144 \\ 7199 & -392.29 & 2.38 & -2.39 & 73.148 \\ 1069 & -392.57 & 2.41 & -1.76 & 73.150 \\ 7198 & -392.78 & 2.41 & -1.98 & 73.151 \\ 7197 & -393.02 & 2.46 & -2.13 & 73.153 \\ 7196 & -393.29 & 2.45 & -2.02 & 73.155 \\ 1068 & -393.53 & 2.58 & -1.57 & 73.156 \\ 7195 & -393.76 & 2.38 & -2.10 & 73.158 \\ 7194 & -394.28 & 2.41 & -2.12 & 73.162 \\ 1067 & -394.54 & 2.31 & -3.08 & 73.163 \\ 7193 & -394.76 & 2.40 & -2.29 & 73.165 \\ 7192 & -395.19 & 2.41 & -2.50 & 73.168 \\ 1066 & -395.4 & 2.44 & -1.95 & 73.169 \\ 7191 & -395.65 & 2.41 & -1.90 & 73.171 \\ 7190 & -395.88 & 2.32 & -2.09 & 73.172 \\ 7189 & -396.13 & 2.43 & -1.88 & 73.174 \\ 1065 & -396.37 & 2.51 & -1.45 & 73.176 \\ 7188 & -396.49 & 2.41 & -1.56 & 73.177 \\ 7187 & -397.16 & 2.39 & -1.79 & 73.181\end{array}$




$\begin{array}{lrlll}1064 & -397.3 & 2.41 & -2.00 & 73.182 \\ 7186 & -397.52 & 2.29 & -2.02 & 73.184 \\ 7185 & -397.78 & 2.26 & -1.82 & 73.185 \\ 7184 & -398.15 & 2.29 & -2.11 & 73.188 \\ 1063 & -398.35 & 2.28 & -1.89 & 73.189 \\ 7183 & -398.44 & 2.28 & -1.96 & 73.190 \\ 7182 & -398.69 & 2.27 & -1.75 & 73.191 \\ 1062 & -399.13 & 2.27 & -2.29 & 73.194 \\ 7181 & -399.75 & 2.27 & -1.66 & 73.199 \\ 1061 & -400 & 2.41 & -1.81 & 73.200 \\ 7180 & -400.3 & 2.32 & -1.66 & 73.202 \\ 7179 & -400.54 & 2.29 & -1.58 & 73.204 \\ 1060 & -401.06 & 2.29 & -1.57 & 73.208 \\ 7178 & -401.25 & 2.30 & -1.93 & 73.209 \\ 7177 & -401.77 & 2.21 & -1.76 & 73.212 \\ 1059 & -401.99 & 2.24 & -2.03 & 73.214 \\ 7176 & -402.54 & 2.30 & -1.91 & 73.218 \\ 1058 & -402.9 & 2.41 & -1.94 & 73.220 \\ 7175 & -403.16 & 2.37 & -1.74 & 73.222 \\ 7174 & -403.65 & 2.37 & -1.64 & 73.225 \\ 1057 & -403.85 & 2.34 & -1.84 & 73.227 \\ 7173 & -404.16 & 2.35 & -1.89 & 73.229 \\ 7172 & -404.5 & 2.20 & -1.92 & 73.231 \\ 1056 & -404.76 & 2.38 & -1.97 & 73.233 \\ 7171 & -405.18 & 2.32 & -1.82 & 73.236 \\ 7170 & -405.6 & 2.31 & -1.68 & 73.238 \\ 1055 & -405.77 & 2.41 & -1.92 & 73.240 \\ 7169 & -405.89 & 2.38 & -1.73 & 73.240 \\ 7168 & -406.32 & 2.35 & -1.89 & 73.243 \\ 1054 & -406.68 & 2.47 & -1.53 & 73.246 \\ 7167 & -406.97 & 2.45 & -1.70 & 73.248 \\ 7166 & -407.25 & 2.38 & -1.50 & 73.250 \\ 1053 & -407.71 & 2.58 & -1.65 & 73.253 \\ 7165 & -408.12 & 2.40 & -1.68 & 73.256 \\ 7164 & -408.34 & 2.48 & -1.72 & 73.257 \\ 1052 & -408.57 & 2.32 & -2.04 & 73.259 \\ 7163 & -408.8 & 2.38 & -1.57 & 73.260 \\ 7162 & -409.05 & 2.33 & -1.68 & 73.262 \\ 1051 & -409.45 & 2.52 & -1.64 & 73.265 \\ 7160 & -409.95 & 2.30 & -1.82 & 73.268 \\ 7158 & -410.02 & 2.51 & -1.92 & 73.269 \\ 7157 & -410.27 & 2.40 & -1.70 & 73.270 \\ 1050 & -410.42 & 2.37 & -1.94 & 73.271 \\ 7156 & -410.45 & 2.38 & -1.75 & 73.271 \\ 7154 & -410.77 & 2.18 & -1.94 & 73.274 \\ 7153 & -410.92 & 2.25 & -1.88 & 73.275 \\ 7151 & -411.21 & 2.26 & -1.66 & 73.277 \\ 1049 & -411.34 & 2.43 & -1.83 & 73.277\end{array}$




$\begin{array}{lllll}7150 & -411.39 & 2.29 & -1.88 & 73.278 \\ 7149 & -411.71 & 2.43 & -1.92 & 73.280 \\ 7148 & -411.95 & 2.31 & -2.23 & 73.284 \\ 1048 & -412.23 & 2.22 & -1.97 & 73.288 \\ 7146 & -412.33 & 2.22 & -1.82 & 73.289 \\ 7145 & -412.59 & 2.29 & -1.99 & 73.293 \\ 7143 & -412.82 & 2.20 & -1.99 & 73.297 \\ 7142 & -412.98 & 2.32 & -1.90 & 73.299 \\ 1047 & -413.02 & 2.24 & -2.02 & 73.300 \\ 7141 & -413.61 & 2.33 & -2.05 & 73.309 \\ 7140 & -413.91 & 2.20 & -1.78 & 73.313 \\ 1046 & -413.98 & 2.22 & -1.96 & 73.315 \\ 7139 & -414.12 & 2.15 & -1.88 & 73.317 \\ 7138 & -414.37 & 2.15 & -2.00 & 73.320 \\ 1045 & -414.49 & 2.21 & -1.89 & 73.322 \\ 7137 & -414.62 & 2.16 & -2.18 & 73.324 \\ 7136 & -414.88 & 2.23 & -1.91 & 73.328 \\ 7135 & -415.08 & 2.18 & -2.13 & 73.331 \\ 7134 & -415.34 & 2.22 & -2.21 & 73.335 \\ 7133 & -415.59 & 2.17 & -1.61 & 73.339 \\ 1044 & -415.78 & 2.20 & -1.93 & 73.342 \\ 7132 & -416.06 & 2.12 & -1.90 & 73.346 \\ 7131 & -416.31 & 2.22 & -2.07 & 73.350 \\ 7130 & -416.56 & 2.18 & -2.03 & 73.354 \\ 1043 & -416.75 & 2.19 & -1.98 & 73.357 \\ 7129 & -416.99 & 2.23 & -2.07 & 73.360 \\ 7128 & -417.24 & 2.14 & -1.95 & 73.364 \\ 7127 & -417.49 & 2.27 & -2.02 & 73.368 \\ 1042 & -417.72 & 2.16 & -2.10 & 73.371 \\ 7126 & -417.96 & 2.21 & -1.77 & 73.375 \\ 7125 & -418.2 & 2.20 & -2.21 & 73.379 \\ 7124 & -418.46 & 2.12 & -1.91 & 73.383 \\ 1041 & -418.65 & 2.25 & -2.09 & 73.386 \\ 7123 & -418.9 & 2.23 & -1.99 & 73.389 \\ 7122 & -419.15 & 2.34 & -1.84 & 73.393 \\ 7121 & -419.42 & 2.23 & -1.91 & 73.397 \\ 1040 & -419.62 & 2.26 & -1.77 & 73.400 \\ 7120 & -419.72 & 2.14 & -2.43 & 73.402 \\ 7119 & -419.99 & 2.20 & -2.32 & 73.406 \\ 7118 & -420.24 & 2.23 & -1.74 & 73.410 \\ 7117 & -420.49 & 2.32 & -1.86 & 73.414 \\ 1039 & -420.67 & 2.23 & -1.91 & 73.416 \\ 7116 & -420.82 & 2.17 & -2.12 & 73.419 \\ 7115 & -421.08 & 2.21 & -1.82 & 73.423 \\ 1038 & -421.53 & 2.21 & -2.03 & 73.429 \\ 7113 & -421.83 & 2.26 & -1.68 & 73.434 \\ 7112 & -422.09 & 2.34 & -1.73 & 73.438 \\ 7111 & -422.35 & 2.22 & -1.75 & 73.442\end{array}$




$\begin{array}{lrlll}1037 & -422.54 & 2.12 & -1.95 & 73.445 \\ 7110 & -422.82 & 2.21 & -1.71 & 73.449 \\ 7109 & -422.96 & 2.20 & -1.89 & 73.451 \\ 7108 & -423.3 & 2.19 & -1.53 & 73.456 \\ 1036 & -423.44 & 2.16 & -1.90 & 73.459 \\ 7107 & -423.64 & 2.18 & -1.86 & 73.462 \\ 7105 & -424.14 & 2.15 & -1.81 & 73.469 \\ 1035 & -424.3 & 2.18 & -1.79 & 73.472 \\ 7104 & -424.52 & 2.15 & -1.92 & 73.475 \\ 7103 & -424.77 & 2.17 & -1.73 & 73.479 \\ 7102 & -425.02 & 2.22 & -2.01 & 73.483 \\ 1034 & -425.24 & 2.28 & -1.92 & 73.486 \\ 7101 & -425.5 & 2.13 & -1.75 & 73.490 \\ 7100 & -425.75 & 2.21 & -1.94 & 73.494 \\ 7099 & -426.02 & 2.23 & -1.89 & 73.498 \\ 1033 & -426.12 & 2.22 & -1.75 & 73.499 \\ 7098 & -426.42 & 2.33 & -1.88 & 73.504 \\ 7097 & -426.68 & 2.17 & -1.90 & 73.508 \\ 7096 & -426.92 & 2.18 & -1.95 & 73.512 \\ 1032 & -427.11 & 2.26 & -1.85 & 73.514 \\ 7095 & -427.42 & 2.30 & -1.75 & 73.519 \\ 7094 & -427.67 & 2.20 & -1.62 & 73.523 \\ 7093 & -427.92 & 2.23 & -1.84 & 73.527 \\ 1031 & -428.03 & 2.25 & -1.62 & 73.528 \\ 5101 & -428.24 & 2.31 & -1.58 & 73.532 \\ 5102 & -428.27 & 2.23 & -1.65 & 73.532 \\ 5103 & -428.3 & 2.33 & -1.71 & 73.533 \\ 5104 & -428.32 & 2.32 & -1.69 & 73.533 \\ 5105 & -428.37 & 2.24 & -1.70 & 73.534 \\ 5106 & -428.42 & 2.25 & -1.63 & 73.534 \\ 7092 & -428.42 & 2.29 & -1.72 & 73.534 \\ 5107 & -428.48 & 2.32 & -1.67 & 73.535 \\ 5108 & -428.53 & 2.20 & -1.88 & 73.536 \\ 5109 & -428.58 & 2.26 & -1.81 & 73.537 \\ 5110 & -428.67 & 2.34 & -1.59 & 73.538 \\ 7091 & -428.68 & 2.26 & -1.35 & 73.538 \\ 5111 & -428.72 & 2.20 & -1.62 & 73.539 \\ 5112 & -428.77 & 2.21 & -1.88 & 73.540 \\ 5113 & -428.87 & 2.25 & -1.59 & 73.541 \\ 7090 & -428.92 & 2.36 & -1.65 & 73.542 \\ 5114 & -428.93 & 2.28 & -1.91 & 73.542 \\ 1030 & -428.94 & 2.34 & -1.92 & 73.542 \\ 5115 & -428.98 & 2.26 & -2.07 & 73.543 \\ 5120 & -429.03 & 2.29 & -1.98 & 73.544 \\ 5121 & -429.06 & 2.27 & -1.98 & 73.544 \\ 5122 & -429.1 & 2.27 & -2.01 & 73.545 \\ 5123 & -429.12 & 2.29 & -1.54 & 73.545 \\ 5125 & -429.14 & 2.30 & -1.56 & 73.545\end{array}$




$\begin{array}{lrlll}5126 & -429.17 & 2.43 & -1.81 & 73.546 \\ 5127 & -429.21 & 2.37 & -1.96 & 73.546 \\ 7089 & -429.25 & 2.33 & -1.64 & 73.547 \\ 5128 & -429.26 & 2.28 & -1.76 & 73.547 \\ 5129 & -429.29 & 2.30 & -1.67 & 73.548 \\ 5130 & -429.33 & 2.33 & -1.84 & 73.548 \\ 5131 & -429.39 & 2.39 & -1.84 & 73.549 \\ 5132 & -429.49 & 2.42 & -1.76 & 73.551 \\ 7088 & -429.5 & 2.29 & -1.76 & 73.551 \\ 5133 & -429.53 & 2.38 & -1.90 & 73.551 \\ 5134 & -429.58 & 2.43 & -1.74 & 73.552 \\ 5135 & -429.63 & 2.36 & -1.92 & 73.553 \\ 5136 & -429.68 & 2.37 & -1.89 & 73.554 \\ 1029 & -429.71 & 2.41 & -1.98 & 73.554 \\ 5137 & -429.73 & 2.38 & -1.73 & 73.554 \\ 5138 & -429.77 & 2.40 & -1.75 & 73.555 \\ 5139 & -429.81 & 2.45 & -1.77 & 73.556 \\ 5140 & -429.84 & 2.39 & -1.78 & 73.556 \\ 5141 & -429.89 & 2.44 & -1.62 & 73.557 \\ 5163 & -429.99 & 2.38 & -1.86 & 73.558 \\ 7087 & -430 & 2.44 & -1.78 & 73.558 \\ 5162 & -430.05 & 2.35 & -1.95 & 73.559 \\ 5161 & -430.09 & 2.38 & -2.02 & 73.560 \\ 5160 & -430.21 & 2.39 & -1.88 & 73.562 \\ 7086 & -430.25 & 2.37 & -1.75 & 73.562 \\ 5159 & -430.26 & 2.43 & -2.04 & 73.562 \\ 5158 & -430.33 & 2.43 & -1.96 & 73.563 \\ 5157 & -430.36 & 2.40 & -1.59 & 73.564 \\ 5156 & -430.42 & 2.34 & -1.66 & 73.565 \\ 5152 & -430.47 & 2.46 & -1.60 & 73.566 \\ 5151 & -430.49 & 2.24 & -1.87 & 73.566 \\ 7085 & -430.5 & 2.37 & -1.78 & 73.566 \\ 5150 & -430.51 & 2.29 & -1.86 & 73.566 \\ 5149 & -430.55 & 2.32 & -1.95 & 73.567 \\ 5148 & -430.6 & 2.31 & -1.79 & 73.568 \\ 5147 & -430.69 & 2.24 & -1.66 & 73.569 \\ 1028 & -430.72 & 2.30 & -1.70 & 73.569 \\ 5146 & -430.72 & 2.28 & -1.65 & 73.569 \\ 7084 & -430.75 & 2.29 & -1.68 & 73.570 \\ 5145 & -430.77 & 2.28 & -1.65 & 73.570 \\ 5144 & -430.83 & 2.33 & -1.88 & 73.572 \\ 5143 & -430.88 & 2.33 & -2.05 & 73.574 \\ 7083 & -431.06 & 2.37 & -1.48 & 73.580 \\ 7082 & -431.33 & 2.38 & -1.76 & 73.588 \\ 7081 & -431.5 & 2.40 & -1.72 & 73.594 \\ 1027 & -431.7 & 2.32 & -1.76 & 73.600 \\ 7080 & -431.83 & 2.29 & -1.91 & 73.604 \\ 7078 & -432.3 & 2.24 & -1.50 & 73.619\end{array}$




$\begin{array}{lllll}1026 & -432.55 & 2.29 & -1.70 & 73.627 \\ 7077 & -432.64 & 2.37 & -1.69 & 73.630 \\ 7076 & -432.88 & 2.27 & -1.81 & 73.637 \\ 7075 & -433.13 & 2.28 & -1.75 & 73.645 \\ 1025 & -433.47 & 2.40 & -1.42 & 73.656 \\ 7073 & -433.72 & 2.25 & -1.64 & 73.664 \\ 7072 & -433.97 & 2.29 & -1.57 & 73.672 \\ 7071 & -434.22 & 2.35 & -1.57 & 73.680 \\ 1024 & -434.56 & 2.28 & -1.84 & 73.691 \\ 7070 & -434.69 & 2.24 & -1.65 & 73.695 \\ 7069 & -434.95 & 2.20 & -1.55 & 73.703 \\ 7068 & -435.11 & 2.20 & -1.54 & 73.708 \\ 1023 & -435.42 & 2.28 & -1.82 & 73.718 \\ 7067 & -435.63 & 2.23 & -1.67 & 73.725 \\ 7066 & -435.87 & 2.27 & -1.67 & 73.733 \\ 7065 & -436.13 & 2.23 & -1.55 & 73.741 \\ 1022 & -436.35 & 2.30 & -1.46 & 73.748 \\ 7064 & -436.54 & 2.26 & -1.64 & 73.754 \\ 7063 & -436.8 & 2.24 & -1.45 & 73.762 \\ 7062 & -437.05 & 2.32 & -1.44 & 73.770 \\ 1021 & -437.29 & 2.34 & -1.58 & 73.778 \\ 7061 & -437.58 & 2.25 & -1.33 & 73.787 \\ 7060 & -437.85 & 2.31 & -1.13 & 73.796 \\ 7059 & -438.03 & 2.29 & -1.66 & 73.801 \\ 1020 & -438.28 & 2.22 & -1.69 & 73.809 \\ 7058 & -438.42 & 2.28 & -1.60 & 73.814 \\ 7056 & -438.89 & 2.25 & -1.46 & 73.829 \\ 1019 & -439.18 & 2.21 & -1.69 & 73.838 \\ 7055 & -439.27 & 2.27 & -1.48 & 73.841 \\ 7054 & -439.5 & 2.24 & -1.47 & 73.848 \\ 7053 & -439.76 & 2.44 & -1.53 & 73.856 \\ 7052 & -440.01 & 2.34 & -1.62 & 73.864 \\ 1018 & -440.22 & 2.26 & -1.68 & 73.871 \\ 7051 & -440.43 & 2.20 & -1.41 & 73.878 \\ 7050 & -440.67 & 2.24 & -1.49 & 73.885 \\ 7049 & -440.93 & 2.24 & -1.31 & 73.894 \\ 1017 & -441.13 & 2.20 & -1.47 & 73.900 \\ 7048 & -441.31 & 2.28 & -1.57 & 73.906 \\ 7047 & -441.56 & 2.30 & -1.54 & 73.914 \\ 7046 & -441.81 & 2.36 & -1.51 & 73.922 \\ 1016 & -441.95 & 2.32 & -1.69 & 73.926 \\ 7045 & -442.32 & 2.28 & -1.59 & 73.938 \\ 7044 & -442.57 & 2.22 & -1.71 & 73.946 \\ 7043 & -442.83 & 2.32 & -1.89 & 73.954 \\ 1015 & -442.96 & 2.23 & -1.69 & 73.958 \\ 7042 & -443.23 & 2.24 & -1.71 & 73.967 \\ 7041 & -443.49 & 2.23 & -1.46 & 73.975 \\ 1014 & -443.69 & 2.31 & -1.42 & 73.981\end{array}$




\begin{tabular}{|c|c|c|c|c|}
\hline 7040 & -443.81 & 2.27 & -1.51 & 73.985 \\
\hline 7039 & -444.11 & 2.30 & -1.76 & 73.995 \\
\hline 7038 & -444.36 & 2.29 & -1.23 & 74.003 \\
\hline 7037 & -444.61 & 2.27 & -1.44 & 74.011 \\
\hline 1013 & -444.81 & 2.31 & -1.66 & 74.017 \\
\hline 7036 & -445.02 & 2.34 & -1.47 & 74.024 \\
\hline 7035 & -445.28 & 2.21 & -1.48 & 74.032 \\
\hline 7034 & -445.53 & 2.23 & -1.46 & 74.040 \\
\hline 1012 & -445.75 & 2.25 & -1.43 & 74.047 \\
\hline 7033 & -445.94 & 2.13 & -1.35 & 74.053 \\
\hline 7032 & -446.21 & 2.11 & -1.48 & 74.062 \\
\hline 7031 & -446.45 & 2.16 & -1.47 & 74.069 \\
\hline 1011 & -446.67 & 2.13 & -1.35 & 74.076 \\
\hline 7030 & -447.04 & 2.18 & -1.45 & 74.088 \\
\hline 7029 & -447.29 & 2.11 & -1.04 & 74.096 \\
\hline 7028 & -447.54 & 2.31 & -1.28 & 74.104 \\
\hline 1010 & -447.65 & 2.06 & -1.67 & 74.107 \\
\hline 7027 & -447.94 & 2.35 & -1.47 & 74.117 \\
\hline 7025 & -448.43 & 2.22 & -1.33 & 74.132 \\
\hline 1009 & -448.59 & 2.37 & -1.55 & 74.137 \\
\hline 7024 & -448.9 & 2.09 & -1.64 & 74.147 \\
\hline 7023 & -449.14 & 2.16 & -1.41 & 74.155 \\
\hline 7022 & -449.39 & 2.20 & -1.37 & 74.163 \\
\hline 1008 & -449.57 & 2.16 & -1.48 & 74.169 \\
\hline 7021 & -449.77 & 2.19 & -1.55 & 74.175 \\
\hline 7020 & -449.98 & 2.22 & -1.65 & 74.182 \\
\hline 7019 & -450.23 & 2.14 & -1.59 & 74.190 \\
\hline 1007 & -450.48 & 2.17 & -1.85 & 74.198 \\
\hline 7018 & -450.7 & 2.22 & -1.75 & 74.205 \\
\hline 7017 & -450.95 & 2.21 & -1.66 & 74.212 \\
\hline 7016 & -451.2 & 2.13 & -1.63 & 74.220 \\
\hline 1006 & -451.44 & 2.14 & -1.74 & 74.228 \\
\hline 7015 & -451.74 & 2.22 & -1.54 & 74.238 \\
\hline 7014 & -451.99 & 2.23 & -1.70 & 74.246 \\
\hline 7013 & -452.18 & 2.14 & -1.65 & 74.252 \\
\hline 1005 & -452.39 & 2.25 & -1.80 & 74.258 \\
\hline 7012 & -452.64 & 2.26 & -1.59 & 74.266 \\
\hline 7011 & -452.88 & 2.21 & -1.35 & 74.274 \\
\hline 7010 & -453.07 & 2.25 & -1.54 & 74.280 \\
\hline 1004 & -453.29 & 2.23 & -1.41 & 74.287 \\
\hline 7009 & -453.52 & 2.29 & -1.71 & 74.294 \\
\hline 7008 & -453.77 & 2.24 & -1.68 & 74.302 \\
\hline 5009 & -453.8 & 2.13 & -0.40 & 74.303 \\
\hline 7007 & -454.03 & 2.23 & -1.65 & 74.311 \\
\hline 1003 & -454.21 & 2.26 & -1.60 & 74.316 \\
\hline 5030 & -454.39 & 2.17 & -0.52 & 74.322 \\
\hline 7006 & -454.44 & 2.21 & -1.59 & 74.324 \\
\hline 7005 & -454.69 & 2.13 & -1.51 & 74.332 \\
\hline
\end{tabular}




$\begin{array}{lllll}7004 & -454.94 & 2.36 & -1.56 & 74.339 \\ 1002 & -455.12 & 2.27 & -1.47 & 74.345 \\ 7003 & -455.29 & 2.18 & -1.47 & 74.351 \\ 7002 & -455.53 & 2.34 & -1.61 & 74.358 \\ 5008 & -455.71 & 2.27 & -0.33 & 74.364 \\ 7001 & -455.78 & 2.30 & -1.44 & 74.366 \\ 1001 & -455.95 & 2.17 & -1.54 & 74.372 \\ 5007 & -456.19 & 2.25 & -1.62 & 74.379\end{array}$

Table S2: Bulk carbonate carbon and oxygen isotope records of Stevns-1. 